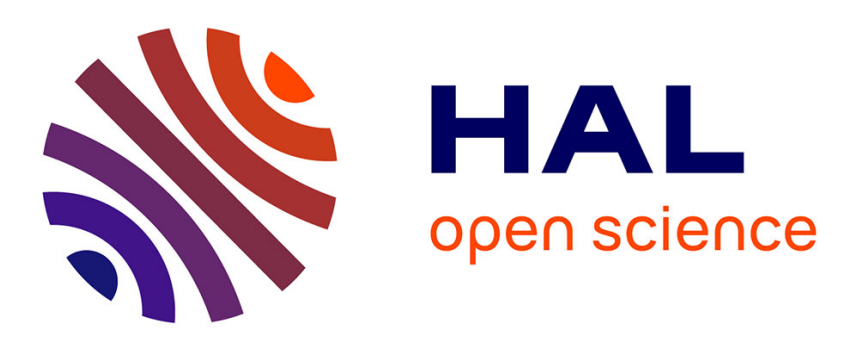

\title{
theory of complex electronic ground state of superconductors: I. Nonadiabatic modification of the Born-Oppenheimer approximation
}

\author{
Pavol Baňacký
}

\section{- To cite this version:}

Pavol Baňacký. theory of complex electronic ground state of superconductors: I. Nonadiabatic modification of the Born-Oppenheimer approximation. Journal of Physics and Chemistry of Solids, 2010, 69 (11), pp.2728. 10.1016/j.jpcs.2008.07.002 . hal-00614335

\section{HAL Id: hal-00614335 \\ https://hal.science/hal-00614335}

Submitted on 11 Aug 2011

HAL is a multi-disciplinary open access archive for the deposit and dissemination of scientific research documents, whether they are published or not. The documents may come from teaching and research institutions in France or abroad, or from public or private research centers.
L'archive ouverte pluridisciplinaire HAL, est destinée au dépôt et à la diffusion de documents scientifiques de niveau recherche, publiés ou non, émanant des établissements d'enseignement et de recherche français ou étrangers, des laboratoires publics ou privés. 


\section{Author's Accepted Manuscript}

Ab initio theory of complex electronic ground state of superconductors: I. Nonadiabatic modification of the Born-Oppenheimer approximation

Pavol Baňacký

PII: $\quad$ S0022-3697(08)00354-5

DOI: doi:10.1016/j.jpcs.2008.07.002

Reference: PCS 5657

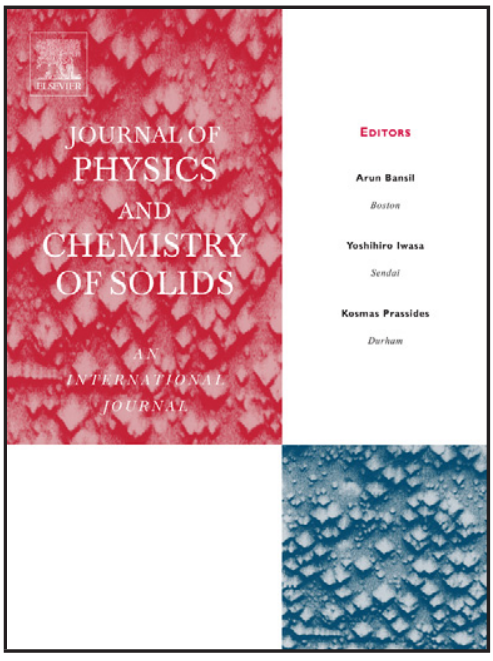

www.elsevier.com/locate/jpcs

To appear in: $\quad$ Journal of Physics and Chemistry of Solids

Received date: $\quad 7$ December 2007

Revised date: $\quad 17$ June 2008

Accepted date: $\quad 3$ July 2008

Cite this article as: Pavol Baňacký, Ab initio theory of complex electronic ground state of superconductors: I. Nonadiabatic modification of the Born-Oppenheimer approximation, Journal of Physics and Chemistry of Solids (2008), doi:10.1016/j.jpcs.2008.07.002

This is a PDF file of an unedited manuscript that has been accepted for publication. As a service to our customers we are providing this early version of the manuscript. The manuscript will undergo copyediting, typesetting, and review of the resulting galley proof before it is published in its final citable form. Please note that during the production process errors may be discovered which could affect the content, and all legal disclaimers that apply to the journal pertain. 


\title{
$A b$ initio THEORY OF COMPLEX ELECTRONIC GROUND STATE OF SUPERCONDUCTORS: I. Nonadiabatic modification of the Born-Oppenheimer approximation
}

\author{
Pavol Baňacký \\ Faculty of Natural Science, Institute of Chemistry, Chemical Physics division, Comenius \\ University, Mlynská dolina CH2, 84215 Bratislava, Slovakia \\ and \\ S-Tech a.s., Dubravská cesta 9, 84105 Bratislava, Slovakia
}

\begin{abstract}
The ARPES of high- $T_{c}$ cuprates and theoretical results of low-Fermi energy band structure fluctuation for different groups of superconductors indicate that electron coupling to pertinent phonon modes drive system from adiabatic into antiadiabatic state $\left(\omega>E_{F}\right)$. At these circumstances, not only Migdal-Eliashberg approximation is not valid, but basic adiabatic Born-Oppenheimer approximation (BOA) does not hold. At these circumstances, electronic structure has to be studied as explicitly dependent on instantaneous nuclear coordinates $Q$ as well as on instantaneous nuclear momenta $P$.

In the present paper - part I, it has been shown that $Q, P$-dependent modification of the BOA for ground electronic state can be derived by sequence of canonical transformations of the basis functions. The effect of nuclear coordinates and momenta on electronic structure is presented in the form of corrections to zero, one and two-particle terms of clamped nuclear Hamiltonian. In the antiadiabatic state, correction to electronic ground state energy (zero-particle term correction) is negative and system can be stabilized in the antiadiabatic state at distorted geometry with respect to adiabatic equilibrium structure and gap in one-particle spectrum of quasi-continuum states at Fermi level can be opened. Stabilization effect is solely the consequence of nuclear dynamics $(P)$ that is crucial in antiadiabatic state. It has been shown that nuclear dynamics also increases electron correlation until system at nuclear motion remains in a bound state. Corresponding corrections to electronic wave function are also specified.

On the other hand, when system remains at vibration motion of nuclei in adiabatic state, the influence of nuclear dynamics ( $P$-dependence) is negligible. In this case, all basic effects are covered through nuclear coordinates ( $Q$-dependence) within the adiabatic BOA and standard results of solid state (or molecular) physics are recovered.

The electronic ground state of superconductors is studied in the subsequent part - II.
\end{abstract}

PACS number(s): 71.10.-w, 74.25.Jb, 74.20.Mn, 74.72.-h

Keywords: A. superconductors; C. ab initio calculations; D. electronic structure; lattice dynamics 


\section{Introduction}

High value of critical temperature $\left(T_{c}\right)$ in the group of cuprate superconductors $[1,2]$ is a serious challenge for theory even 20 years since discovery of this group of materials. The high value of $T_{c}$ should be related to corresponding high value of electron-phonon (EP) coupling constant, $\lambda \geq 1$. The standard, generally accepted EP-based theories of superconductivity, BCS or BCS-like theories [3-5], assume validity of the Migdal theorem and Eliashberg restriction [4,5] (ME approximation). The first is related to validity of the condition $\omega \lambda / E_{F}$ $<<1$ and the second one restricts the validity only for $\lambda \leq 1$. Expressed explicitly, BCS-like theories are valid only for adiabatic systems that obey the Born-Oppenheimer approximation (BOA): $\omega / E_{F}<<1$. Only at these circumstances, separation of electronic and nuclear motion is well justified and one can study electrons and nuclei as two statistically independent fields with mutual interaction that corrects the electronic energy and renormalizes the phonon frequencies. However, it has been shown [6] by parameter-free estimate for high- $T_{c}$ cuprates and also for fullerides and $\mathrm{MgB}_{2}$ that Fermi energy $\left(\mathrm{E}_{\mathrm{F}}\right)$ of this group of materials is on the same energy scale as it corresponds to the energy of the relevant optical phonon modes, $\mathrm{E}_{\mathrm{F}} \approx$ $\omega$. Problems with EP interactions within the ME scenario gave rise to variety of non-phonon coupling models with stress focused on the role of electron correlations at the transition to superconducting (SC) state. The EP interactions, which have been accepted to be responsible for electron pairing that drive transition into superconducting state for classical low- $\mathrm{T}_{\mathrm{C}}$ superconductors, have became nearly abandoned and considered to be rather harmful for superconductivity in high- $T_{c}$ cuprates [7]. Some aspects of $d-$ wave superconductivity can be described within the models of strongly correlated electrons, e.g. Hubbard - like or $t-J$ models (e.g. [8-11]), without explicit account for EP interactions (see also variation theory [12]). The underlying light motive behind the electron correlation treatments has been to understand the phase diagram of high- $\mathrm{T}_{\mathrm{c}}$ cuprates, i.e. the doping process. Introduction of charge carriers (holes or electrons) into the parent antiferomagnetic insulator that causes transition to superconductor (or metal) has been generally accepted to be a universal feature of high- $\mathrm{T}_{\mathrm{c}}$ cuprates and believed to be a matter intimately related to microscopic mechanism of superconductivity. Recent results of high-resolution ARPES study [13,14] of the wide family of different high- $\mathrm{T}_{\mathrm{c}}$ cuprates have brought surprising direct experimental evidence that it is not doping but an abrupt change (decrease) of the electron velocity near Fermi level, at about $50-80 \mathrm{meV}$, that is the universal feature common to high- $\mathrm{T}_{\mathrm{c}}$ cuprates. Even more important in this respect is formation of T-dependent giant kink on momentum distribution curve close to Fermi level that is present at and below $\mathrm{T}_{\mathrm{c}}$. It has been recorded [15] in the offnodal direction of Bi2223.

These results along with the results of neutron scattering $[16,17]$ indicate that also for high$\mathrm{T}_{\mathrm{c}}$ cuprates EP coupling has to be considered as a crucial element of microscopic mechanism of SC state transition. As soon as low-Fermi energy situation occurs $\left(\omega \leq E_{F}\right)$ one can expect important contribution of nonadiabatic vertex corrections at SC state transition. It is beyond the standard ME approximation and this problem has been studied within the nonadiabatic theory of superconductivity [18a,b, 30]. On the other hand, as the ARPES results indicate, electron kinetic energy is decreased and importance of proper treatment of electron-electron Coulomb interactions is increased. The competition between Coulomb vs. EP interactions has been intensively studied within the Holstein - Hubbard models [19-23] with both interactions introduced as short-range order. The obtained results are not satisfactory since heavy-mass polarons are formed that yields low values of $\mathrm{T}_{\mathrm{c}}$. It has been improved within the Frohlich Coulomb model [24a] that introduces long-range order repulsion between charge-carriers and also long-range order EP interactions. The results show that there is a narrow window of parameters of Coulomb repulsion $V_{c}$ and $E P$ interactions $E_{P}\left(V_{c} / E_{P}\right)$ resulting in the lightmass bipolarons formation. In this case, according to bipolaron theory of superconductivity 
[24b,c,d], coherent motion of bipolarons represents the supercarrier motion and high $\mathrm{T}_{\mathrm{c}}$ can be reached.

Theoretical studies [25-29] of the dependence of band structure of $\mathrm{MgB}_{2}$ on electron coupling to the $\mathrm{E}_{2 \mathrm{~g}}$ phonon mode $\left(\omega_{E_{2 g}} \approx 0.066 \mathrm{eV}\right.$ ) have revealed even more important new aspect that seems to be crucial for microscopic theory of electronic ground state of superconductors. It has been shown that vibration motion of B-B atoms in the $E_{2 g}$ phonon mode induces periodic fluctuation of the top (analytic critical point - ACP) of one of the $\sigma$ band across Fermi level in the $\Gamma$ point of $1^{\text {st }}$ BZ. Fermi level crossing occurs at nuclear displacement that corresponds to root-mean-square (rms) displacement of vibration zero-point energy $[28,29]$. It means, that due to EP interactions, the Fermi energy (chemical potential of $\sigma$ band electrons) is considerably reduced (from initial value $E_{F} \approx 0.5 \mathrm{eV}$ ) and at the moment when the ACP of the band touches Fermi level, the Fermi energy is reduced to zero, $E_{F} \rightarrow 0$. From the physical stand-point it represents transition of the system from adiabatic $\omega \leq E_{F}$ into intrinsic nonadiabatic $\omega>E_{F}$, or even to strong antiadiabatic state with $\omega>E_{F}$. This effect has crucial theoretical impact. At these circumstances, not only ME approximation is not valid (including impossibility to calculate nonadiabatic vertex corrections [30] that represent off-diagonal corrections to adiabatic ground state), but adiabatic BOA itself does not hold. Low-Fermi energy periodic fluctuation of band structure has been recently reported [31] also for high- $\mathrm{T}_{\mathrm{c}}$ cuprate $\mathrm{YBa}_{2} \mathrm{Cu}_{3} \mathrm{O}_{7}$. This effect is absent in respective nonsuperconducting analogs, e.g. $\mathrm{AlB}_{2}$ and $\mathrm{YBa}_{2} \mathrm{Cu}_{3} \mathrm{O}_{6}[28,31]$.

Transition from adiabatic $\left(\omega<<E_{F}\right)$ into antiadiabatic $\left(\omega>>E_{F}\right)$ state due to EP interactions, seems to be the basic physical effect that is common for superconductors. It means, however, breakdown of the adiabatic BOA, i.e. breakdown of the approximation that is the very basic starting point of many-body theory of solids, including BCS theory as well as models of strongly correlated electrons in case of superconductors. Formulation of selfconsistent theory that will be able to account for breakdown of the BOA has been recently declared [32] to be the most challenging line in research of superconductivity.

On the level of the BOA, the motion of the electrons is a function of the instantaneous nuclear coordinates (usually only parametric dependence is considered), but is not dependent on the instantaneous nuclear momenta (velocities). Nuclear coordinate-dependence modifies nuclear potential energy by so called diagonal BO correction (DBOC) that reflects an influence of small nuclear displacements out-of the equilibrium positions and corrects the electronic energy of clamped nuclear structure. The DBOC enters directly into the potential energy term of nuclear motion (but leaves unchanged the nuclear kinetic energy) and in this way modifies vibration frequencies. The off-diagonal terms of the nuclear part of system Hamiltonian that mix electronic and nuclear motion through the nuclear kinetic energy operator term are neglected and it enables independent diagonalization of electronic and nuclear motion (adiabatic approximation). Neglecting the off-diagonal terms is justified only if these are very small - adiabatic conditions, i.e. if the energy scales of electron and nuclear motion are very different and when it holds $\omega / \mathrm{E}<<1$. If necessary, small contribution of the off-diagonal terms can be calculated by perturbation methods as so called nonadiabatic (vertex) correction to the adiabatic ground state.

Situation for superconductors seems to be substantially different. There is considerable reduction of electron kinetic energy, which for antiadiabatic state results even for dominance of nuclear dynamics $\left(\omega>>E_{F}\right)$ in some region of $k$-space. In this case, it is necessary to study electronic motion as explicitly dependent on the operators of instantaneous nuclear coordinates $Q$ as well as on operators of instantaneous nuclear momenta $P$. It is a new aspect for many-body theory. 
In the present paper - part I, theoretical background of nonadiabatic $P$-dependent modification of the adiabatic BOA is presented along with corresponding sequence of canonical transformations of general nonrelativistic form of system Hamiltonian (molecular or solid state system). It is related to our original formulation of molecular electron-vibration theory on the Hartree-Fock SCF (HF-SCF) level [33]. Solution of the final nonadiabatic form that has been obtained in real space orbital representation is transformed into quasimomentum $k, q$ space representation of solids. As there are some differences in details of Hartree-Fock treatment in solid state theory and in molecular physics, in section II. (Preliminaries) the quasi-particle form of general molecular Hamiltonian on crude-adiabatic and adiabatic level within the HF-SCF solution is presented. In section III, theoretical background of canonical - base transformations of clamped nuclear (crude-adiabatic) Hamiltonian and electronic wave function to adiabatic $Q$-dependent level and to nonadiabatic $Q, P$-dependent level (nonadiabatic modification of the BOA) are presented. The results in the following sections are expressed in real space orbital representations as well in quasimomentum space representation of solids. Dependence of electronic energy terms, i.e. corrections to zero-particle term (ground state energy), to one-particle term, including gap opening and to two-particle term, on nuclear coordinates and momenta are presented in section IV. There is supplementary part, Appendix A - C, that presents details to the particular parts in the main text. Electronic ground state of superconductors is studied in the subsequent paper that is the part II to the present theoretical formulation.

\section{Preliminaries}

General form of nonrelativistic Hamiltonian of molecular or solid state system can be written in second quantization formalism as an explicit function of electron $\left(a^{+}, a\right)$ and nuclear $\left(b^{+}, b\right)$ creation and annihilation operators,

$$
\begin{aligned}
& H=T_{N}+T_{e}(r)+V_{e e}(r)+V_{e N}(r, R)+E_{N N}(R)= \\
& =T_{N}(P)+E_{N N}(Q)+\sum_{P Q} h_{P Q}(Q) a_{P}^{+} a_{Q}+\frac{1}{2} \sum_{P Q R S} v_{P Q R S}^{0} a_{P}^{+} a_{Q}^{+} a_{S} a_{R}
\end{aligned}
$$

The nuclear potential energy $E_{N N}$ and one-electron core term $h_{P Q}$ (electron kinetic energy plus electron-nuclear coulomb attraction term) are functions of the nuclear coordinate $Q_{r}$ operators (normal modes nuclear displacements out-of fixed nuclear geometry $R_{0}$ ) and nuclear kinetic energy $T_{N}$ is a quadratic function of the corresponding nuclear momenta operators $P_{r}$. The letters $Q, P$ which stand for nuclear coordinate and momentum operators and $\mathrm{R}, r$ that denote nuclear and electron coordinates should be distinguished from subscripts in matrix elements where these letters indicate index of the orbitals (e.g. $\left\langle\varphi_{P}|A| \varphi_{Q}\right\rangle=A_{P Q}, v_{P Q R S}^{0}, \ldots$ ) and phonon mode $r\left(\omega_{\mathrm{r}}, \mathrm{Q}_{\mathrm{r}}, ..\right)$, respectively. In order to keep open possibility of application for real as well for complex wave functions, it is assumed that besides vibration mode $r$ there is also corresponding mode $\breve{r}\left(\omega_{r}=\omega_{\breve{r}}\right)$ to which in quasi-momentum space of solids corresponds wave vectors $q$ and $(-q)$. In case of molecular systems with real wave functions holds $r \equiv \breve{r}$. For solids, in electronic quasi-momentum $k$-space representation, it is assumed that to spinorbital $\varphi_{R}(k, \sigma)$ corresponds complex conjugate spinorbital $\breve{\varphi}_{R}(-k, \pm \sigma)$. Also in this case, for real wave functions of molecular systems $\breve{\varphi}_{R} \equiv \varphi_{R}$.

For nuclear coordinate and momentum operators hold:

$$
\begin{aligned}
& Q_{r}=\left(b_{r}+b_{\breve{r}}^{+}\right), Q_{\breve{r}}=\left(b_{\breve{r}}+b_{r}^{+}\right), Q_{r}^{+}=\left(b_{r}^{+}+b_{\breve{r}}\right)=Q_{\breve{r}} \\
& P_{r}=\left(b_{r}-b_{\breve{r}}^{+}\right), P_{\breve{r}}=\left(b_{\breve{r}}-b_{r}^{+}\right), P_{r}^{+}=\left(b_{r}^{+}-b_{\breve{r}}\right)=-P_{\breve{r}}
\end{aligned}
$$


The $Q$-dependence of terms $E_{N N}(Q)$ and $h_{P Q}(Q)$ in (1) can be expressed through the Taylor's expansion at fixed nuclear configuration $R_{0}$,

$E_{N N}(Q)=E_{N N}^{0}\left(R_{0}\right)+\sum_{j=1}^{\infty} E_{N N}^{(j)}(Q) \quad h_{P Q}(Q)=h_{P Q}^{0}\left(R_{0}\right)+\sum_{i=1}^{\infty} u_{P Q}^{(i)}(Q)$

Term $E_{N N}^{0}\left(R_{0}\right)$ represents potential energy at fixed nuclear configuration $R_{0}, h_{P Q}^{0}\left(R_{0}\right)$ is oneelectron core term at fixed nuclear configuration $R_{0}$ and terms $\left\{u_{P Q}^{(i)}(Q)\right\}$ are related to matrix elements of electron - vibration (phonon) coupling $\left(u_{P Q}^{(i)}\right)$, i.e.

$u_{P Q}^{r}(Q)=\frac{\partial h_{P Q}(Q)}{\partial Q_{r}} Q_{r}=u_{P Q}^{r} Q_{r} ; \quad u_{P Q}^{r \ldots s}(Q)=\frac{\partial h_{P Q}(Q)}{\partial Q_{r} \ldots \partial Q_{s}} Q_{r} \ldots Q_{s}=u_{P Q}^{r \ldots s} Q_{r} \ldots Q_{s}$

Two-electron terms $v_{P Q R S}^{0}$ (electron-electron coulomb repulsion and exchange integrals) do not depend explicitly on the nuclear operators.

It is obvious that the following symmetry relations have to hold,

$h_{P Q}^{0}=h_{Q P}^{0^{*}}, \quad h_{P Q}^{0}=h_{\breve{Q P}}^{0}, \quad v_{P Q R S}^{0}=v_{Q P S R}^{0}=v_{S R Q P}^{0^{*}}=v_{R S P Q}^{0^{*}}, \quad v_{P Q R S}^{0}=v_{\breve{R S P Q}}^{0}$

For coefficients of the Taylor's expansions up to second order, the following identities are required to hold,

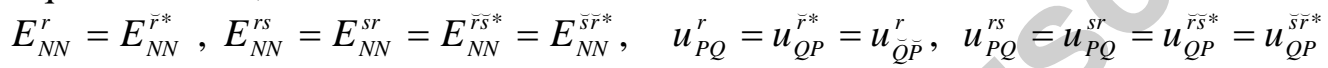

There are several possibilities of approximate solution of this many-body problem. Assumption of validity of the Born-Oppenheimer approximation (BOA) is the basis of these treatments. Since the term BOA and subsequent applications are often understood in a simplistic way, some aspects of the BOA should be specified.

Solution of the Schrödinger equation of many-body system composed of $n_{e}$ electrons and $\mathrm{N}_{\mathrm{n}}$ nuclei (total system), $H \Psi(r, R)=E^{T S} \Psi(r, R)$

with the Hamiltonian (1) and wave function $\Psi(r, R)$, which is a general function of the sets of electron $\{r\}$ and nuclear $\{R\}$ coordinates, is possible in the assumption of validity of the BOA. It was originally formulated [34] by power expansion of potential surface for nuclear motion at equilibrium geometry with respect to displacement and electron/nuclear mass ratio $\left(m_{e} / M_{n}\right)^{1 / 4}$ and reformulated later by Born (see e.g. appendix in [35]) in a more practical form. According to it, the crucial point of the BOA is that the wave function of the total system (6) can be expressed in the factorized form,

$\Psi(r, R)=\sum_{m} \chi_{m}(R) \Phi_{m}(r, R)$

It is a linear combination of known adiabatic electronic wave functions $\left\{\Phi_{m}(r, R)\right\}$ that are the eigenfunctions of clamped nuclear electronic Schrödinger equation,

$H_{e} \Phi_{m}(r, R)=E_{m}^{e} \Phi_{m}(r, R)$

The electronic Hamiltonian in (8) corresponds to fixed nuclear configuration R, $H_{e}=T_{e}+V_{e e}(r)+V_{e N}(r, R)$

Expansion coefficients $\left\{\chi_{m}(R)\right\}$ in (7), regarded as unknown, are nuclear wave functions for nuclear configuration $\mathrm{R}$ with the electronic subsystem in particular adiabatic electronic state $\Phi_{m}(r, R)$.

The Schrödinger equation of the total system (6) for electronic state $\Phi_{n}(r, R)$ with respect to wave function (7) can be written in the form,

$\left\{T_{N}+E_{n}^{t e}(R)-E^{T S}(R)\right\} \chi_{n}(R)=\sum_{m} \Lambda_{n m}(R) \chi_{m}(R)$ 
with the rhs term $\Lambda_{n m}(R)$,

$$
\begin{aligned}
\Lambda_{n m}(R)=A_{n m}(R)-B_{n m}(R)= & \sum_{j} \frac{\hbar^{2}}{M_{j}} \int \Phi_{n}^{*}\left(r, R_{j}\right) \frac{\partial}{\partial R_{j}} \Phi_{m}\left(r, R_{j}\right) d r \cdot \frac{\partial}{\partial R_{j}}- \\
& -\int \Phi_{n}^{*}(r, R) T_{N}(R) \Phi_{m}(r, R) d r
\end{aligned}
$$

Term $E_{n}^{\text {te }}$ in (10),

$$
E_{n}^{t e}(R)=\left(E_{n}^{e}(R)+E_{N N}(R)\right)
$$

is total adiabatic electronic energy, i.e. adiabatic electronic energy plus nuclear Coulomb repulsion at nuclear configuration R. Until the Born approach (7) is valid, equation (10) is exact and it still describes coupled motion of electrons and nuclei through the term (11) $\Lambda_{n m}(R)$, which represents possibility of transitions between different adiabatic electronic states $\Phi_{n}(r, R) \leftrightarrow \Phi_{m}(r, R)$, due to nuclear motion (R - dependence). If such transitions are forbidden from the symmetry reasons, or if there is physically reasonably justified assumption that contributions of such transitions are negligibly small, then one can omit the rhs term and equation (10) can be written in the diagonal form,

$\left\{T_{N}+\left(E_{n}^{t e}(R)+B_{n n}(R)\right)-E_{n, v}^{T S}(R)\right\} \chi_{n, v}(R)=0$

Since $A_{n n}=0$, the only non-zero diagonal contribution of the $\Lambda$ term in (13) is $B_{n n}$,

$$
\begin{aligned}
B_{n n}(R) & =\int \Phi_{n}^{*}(r, R) \hat{T}_{N}(R) \Phi_{n}(r, R) d r=\sum_{n}^{N}-\frac{\hbar^{2}}{2 M_{n}} \int \Phi_{n}^{*}(r, R) \nabla_{n}^{2} \Phi_{n}(r, R) d r= \\
& =\left.\sum_{n \alpha} \frac{\hbar^{2}}{2 M_{n}} \int\left(\frac{\partial \Phi_{n}(r, \vec{R})}{\partial R_{n \alpha}}\right)^{*}\left(\frac{\partial \Phi_{n}(r, \vec{R})}{\partial R_{n \alpha}}\right) d r\right|_{[\mathrm{R}]}
\end{aligned}
$$

Term $B_{n n}$ is the mean-value of the nuclear kinetic energy for adiabatic electronic state $\Phi_{n}(r, R)$ at instantaneous nuclear configuration $\mathrm{R}$ and represents so called adiabatic diagonal Born-Oppenheimer (DBOC) correction to the total adiabatic electronic energy $E_{n}^{t e}(R)$. The equation (13) is then the equation of motion of nuclei and it has the form of Schrödinger equation with Hamiltonian,

$H_{N}(R)=T_{N}+E_{N N}^{e f f}(R)$

The effective-adiabatic potential for nuclear motion $E_{N N}^{\text {eff }}(R)$,

$E_{N N}^{e f f}(R)=E_{n}^{t e}(R)+B_{n n}(R)$

is represented by the total electronic energy (12) which is corrected by mean-value of the nuclear kinetic energy (DBOC) for the particular adiabatic electronic state $\Phi_{n}(r, R)$ - (14). At these circumstances the motion of electrons and nuclei is effectively decoupled and it is possible to realize an independent diagonalization of the electronic Schrödinger equation (8) and nuclear Schrödinger equation (13). In this case, electrons and nuclei of the system behave like two statistically independent sets.

Contribution of the rhs term of equation (10) is assumed to be small and it can be calculated by some approximate way, usually by perturbation theory. Then, problem with the Hamiltonian,

$$
H(R)=H^{0}(R)+H^{\prime}(R)
$$

is studied. Unperturbed part is,

$$
H^{0}(R)=H_{t e}(R)+H_{N}(R)=\left\{(9)+E_{N N}(R)\right\}+(15)
$$

Perturbation is represented by the off-diagonal terms,

$$
H^{\prime}(R)=\hat{\Lambda}_{m n}(R) ; \quad\left(\quad H_{m n}^{\prime}(R)=\int \chi_{m \mu}^{*}(R) \hat{\Lambda}_{m n}(R) \chi_{n v}(R) d R\right) ; \mathrm{m} \neq \mathrm{n}
$$


In practice, physical and/or chemical properties of a many-body system in its ground electronic state $\Phi_{0}(r, R)$ are of the prime interest. In this case, the Born approach (7) is usually restricted to the single term and the total wave function of system is a simple product of the ground state adiabatic electronic wave function $\Phi_{0}(r, R)$ and corresponding nuclear wave function,

$\Psi_{0}(r, R)=\chi_{0, v}(R) \Phi_{0}(r, R)$;

The Born approach in the form (20) is generally called the adiabatic approximation. In a common sense, what is usually understand as the Born-Oppenheimer approximation (or crude-adiabatic) is the adiabatic approximation where the contribution of the DBOC (i.e. $\mathrm{B}_{00}(\mathrm{R})$ term in (16)) is also neglected and wave function (20) is rather of the form, $\Psi_{0}(r, R)=\chi_{0, v}(R) \Phi_{0}\left(r, R_{e q}\right)$

Contributions of the off-diagonal terms $\hat{\Lambda}_{0 m}(R)$ - (19) that are calculated as a small perturbation to the Hamiltonian (17) represent a nonadiabatic correction to the unperturbed adiabatic ground state (see e.g. [36]). The conditions at which the nonadiabatic correction can be expected to be small and the BOA (20, and in general 7) is valid, can be estimated by analysis of the second order contributions to the energy of the total system, $E_{0}^{T S}(R)$, which are small providing that,

$$
\left|\int \chi_{0, v}^{*}(R) \hat{\Lambda}_{0 m}(R) \chi_{m, \mu}(R) d R\right|<<\left|E_{0, v}^{T S}(R)-E_{n, \mu}^{T S}(R)\right|
$$

By expansion, at least up to the quadratic term in a displacement $\mathrm{Q}$ of the effective nuclear potential ( $V_{N N}^{\text {eff }}(R)=E_{0}^{\text {te }}(R)$ ) at equilibrium nuclear geometry $\mathrm{R}_{\mathrm{eq}}$, it can be derived that (22) holds and the BOA $(20,7)$ is valid if the inequality

$\left|E_{0}^{t e}\left(R_{e q}\right)-E_{n}^{t e}\left(R_{e q}\right)\right|>>\hbar \omega_{s}$

is fulfilled for electronic and vibration (phonon) energy spectrum of a system. The meaning of (23) is clear, the electronic frequency spectrum, i.e. the differences between the total electronic energies of the excited electronic states and the ground state energy has to be much greater than vibration (phonon) energy spectrum of the system.

\section{Hierarchy of approximations}

\section{II.1. Crude- adiabatic approximation}

Providing that phonon and electronic energy spectrum are well separated and (23) holds for relevant configuration space $\mathrm{R}$ near to $\mathrm{R}_{\mathrm{eq}}$, crude-adiabatic (clamped nuclear) treatment is justified. In this case, electronic and nuclear part of the Hamiltonian (1) is treated as statistically independent sets.

Electronic Hamiltonian is only parametrically dependent on nuclear configuration, i.e. nuclear geometry is fixed at nuclear configuration $R_{0}$,

$$
H_{e}=E_{N N}^{0}\left(R_{0}\right)+\sum_{P Q} h_{P Q}^{0}\left(R_{0}\right) \bar{a}_{P}^{+} \bar{a}_{Q}+\frac{1}{2} \sum_{P Q R S} v_{P Q R S}^{0} \bar{a}_{P}^{+} \bar{a}_{Q}^{+} \bar{a}_{S} \bar{a}_{R}
$$

Application of the Wick's theorem to the product of creation and annihilation operators yields for particular terms the normal product form (N[...]) with corresponding contractions,

$$
\begin{aligned}
& \sum_{P Q} h_{P Q}^{0} \bar{a}_{P}^{+} \bar{a}_{Q}=\sum_{P Q} h_{P Q}^{0} N\left[\bar{a}_{P}^{+} \bar{a}_{Q}\right]+\sum_{I}^{o c c} h_{I I}^{0} \\
& \sum_{P Q R S} v_{P Q R S}^{0} \bar{a}_{P}^{+} \bar{a}_{Q}^{+} \bar{a}_{S} \bar{a}_{R}=\sum_{P Q R S} v_{P Q R S}^{0} N\left[\bar{a}_{P}^{+} \bar{a}_{Q}^{+} \bar{a}_{S} \bar{a}_{R}\right]+\sum_{P Q}\left(\sum_{I}^{o c c}\left(v_{P I Q I}^{0}+v_{I P I Q}^{0}-v_{P I I Q}^{0}-v_{I P Q I}^{0}\right)\right) N\left[\bar{a}_{P}^{+} \bar{a}_{Q}\right]+ \\
& +\sum_{I J}^{o c c}\left(v_{I J I J}^{0}-v_{I J J I}^{0}\right)
\end{aligned}
$$


At the Wick's theorem application, the renormalized Fermi vacuum $\Phi_{0}$ is introduced and the total set of orthonormal base orbitals $\left(\varphi_{\text {}_{P}}, \varphi_{Q}, ..\right)$ is divided on two distinct groups; the set of occupied $\left(\varphi_{I}, \varphi_{J}, ..\right)$ and set of unoccupied $\left(\varphi_{A}, \varphi_{B}, ..\right)$ spinorbitals.

At this moment, the electronic Hamiltonian (24) can be written in a quasi-particle form as a sum of zero, one and-two particle terms,

$$
H_{e}=H_{(0)}+H_{(1)}+H_{(2)}=\left(E_{N N}^{0}+H_{e}^{0}\right)+H_{(1)}+H_{(2)}=E_{0}^{t e}\left(R_{0}\right)+H_{(c a d)}^{\prime}\left(R_{0}\right)+H_{(c a d)}^{\prime \prime}\left(R_{0}\right)
$$

a/ The scalar quantity, $H_{e}^{0}=H_{(\text {cad })}^{0}\left(R_{0}\right)$, i.e. zero-particle term in $H_{(0)}$, is the result of the operators contractions and has the form,

$$
H_{e}^{0}=\left\langle\Phi_{0}\left|H_{e}\right| \Phi_{0}\right\rangle=\sum_{I}^{o c c} h_{I I}^{0}+\frac{1}{2} \sum_{I J}^{o c c}\left(v_{I J I J}^{0}-v_{I J J I}^{0}\right)=E_{S C F}^{0}\left(R_{0}\right)
$$

This term represents ground state electronic energy calculated by the Hartree-Fock SCF (HFSCF) procedure at fixed nuclear configuration $R_{0}$. Electronic ground state is represented by renormalized Fermi vacuum $\Phi_{0}$. It is an antisymmetric electronic wave function that is expressed in the form of single Slater determinant constituted by lowest laying occupied spinorbitals $\left\{\varphi_{I}\right\}$ of complete orthonormal base $\left\{\varphi_{P}\right\}$,

$\Phi_{0}\left(r, R_{0}\right)=\left|\varphi_{1} \ldots \ldots \ldots \varphi_{I}\right|$

b/ The one-particle term $H_{(1)}=H_{(c a d)}^{\prime}\left(R_{0}\right)$ of the electronic Hamiltonian (26) has the form,

$$
H_{(1)}=\sum_{P Q} F_{P Q} N\left[\bar{a}_{P}^{+} \bar{a}_{Q}\right]
$$

The elements $F_{P Q}$ are calculated as matrix elements of the Hartree-Fock one-particle operator

$$
F\left(R_{0}\right)=h^{0}\left(R_{0}\right)+\sum_{Q}\left(J_{Q}-K_{Q}\right)
$$

Diagonalization of (29), $F_{P Q}=\varepsilon_{P}^{0} \delta_{P Q}$, i.e. solution of electronic Hartree-Fock equations by HF-SCF procedure,

$$
F(R) \varphi_{P}\left(r, R_{0}\right)=\sum_{Q} \varepsilon_{P Q} \varphi_{Q}\left(r, R_{0}\right)
$$

yields set of eigenvalues, i.e. HF-orbital energies

$\varepsilon_{P}^{0}=h_{P P}^{0}+\sum_{Q}\left(v_{P Q P Q}^{0}-v_{P Q Q P}^{0}\right)$

and corresponding set of eigenfunctions $\left\{\varphi_{P}\right\}$ - the orthonormal set of optimized spinorbitals.

It means that one-particle term (29) can always be written in diagonal form and represents set of eigenenergies of system, i.e.

$H_{(1)}=\sum_{P} \varepsilon_{P}^{0} N\left[\bar{a}_{P}^{+} \bar{a}_{P}\right]$

In an approximate way, the one-particle Hamiltonian $H_{(1)}$ represents complete electronic spectrum expressed over occupied and unoccupied - virtual spinorbitals, which are calculated for electronic ground state $\Phi_{0}\left(r, R_{0}\right)$ by the HF-SCF procedure. In particular, $n$-electron “excited" state wave function $\Phi_{h_{n} p_{n}}$ can be constructed by promotion of $n$ electrons from $n$ occupied spinorbitals to $n$ unoccupied spinorbitals (i.e. the same number $n$ of holes $\left(\mathrm{h}_{n}\right)$ and particles $\left(\mathrm{p}_{\mathrm{n}}\right)$ are created). Electronic energy of such excited state can be calculated through HF-eigenenergies that correspond to optimized spinorbitals of the ground state. However, exact treatment of excited states is different and it will be mentioned shortly in section II.2. In terms of the orbital energies (29c), for total electronic ground state energy $E_{0}^{t e}\left(R_{0}\right)$ holds, 


$$
\begin{aligned}
& E_{0}^{t e}\left(R_{0}\right)=E_{N N}^{0}\left(R_{0}\right)+E_{S C F}^{0}\left(R_{0}\right)=E_{N N}^{0}\left(R_{0}\right)+\left(\sum_{I}^{o c c} h_{I I}^{0}+\frac{1}{2} \sum_{I J}^{o c c}\left(v_{I J I J}^{0}-v_{I J J I}^{0}\right)\right)= \\
& =E_{N N}^{0}\left(R_{0}\right)+\frac{1}{2} \sum_{I}^{o c c}\left(\varepsilon_{I}^{0}+h_{I I}^{0}\right)
\end{aligned}
$$

For closed-shell electronic systems within restricted HF approximation, spinorbitals are expressed over spatial orbitals whereas each spatial orbital can be occupied by two electrons with opposite spins, i.e. spinorbitals $\varphi_{P}$ and $\varphi_{P+1}$ are replaced by the same spatial orbital with different spin parts, $\varphi_{P} . \alpha$ and $\varphi_{P} . \beta$. In this case, the HF operator (29a), HF-orbital energies (29c) and electronic energy $(27,30)$ are correspondingly modified,

$$
\begin{aligned}
& F\left(R_{0}\right)=h^{0}\left(R_{0}\right)+\sum_{Q}\left(2 J_{Q}-K_{Q}\right) \\
& \varepsilon_{P}^{0}=h_{P P}^{0}+\sum_{Q}\left(2 v_{P Q P Q}^{0}-v_{P Q Q P}^{0}\right) \\
& H_{e}^{0}=\left\langle\Phi_{0}\left|H_{e}\right| \Phi_{0}\right\rangle=2 \sum_{I}^{o c c} h_{I I}^{0}+\sum_{I J}^{o c c}\left(2 v_{I J I J}^{0}-v_{I J J I}^{0}\right)=E_{S C F}^{0}\left(R_{0}\right) \\
& E_{0}^{t e}\left(R_{0}\right)=E_{N N}^{0}\left(R_{0}\right)+E_{S C F}^{0}\left(R_{0}\right)=E_{N N}^{0}\left(R_{0}\right)+\left(2 \sum_{I}^{o c c} h_{I I}^{0}+\sum_{I J}^{o c c}\left(2 v_{I J I J}^{0}-v_{I J J I}^{0}\right)\right)= \\
& =E_{N N}^{0}\left(R_{0}\right)+\sum_{I}^{o c c}\left(\varepsilon_{I}^{0}+h_{I I}^{0}\right)
\end{aligned}
$$

Total electronic ground state energy of the system reaches the minimum at some equilibrium nuclear configuration $R_{0}=R_{e q}$. Corresponding Slater determinant (28) represents wave function of the electronic ground state at equilibrium nuclear configuration.

c/ The third term of the electronic Hamiltonian (26), i.e. two-particle term has the form,

$$
H_{(2)}=H_{(c a d)}^{\prime \prime}\left(R_{0}\right)=\frac{1}{2} \sum_{P Q R S} v_{P Q R S}^{0} N\left[\bar{a}_{P}^{+} \bar{a}_{Q}^{+} \bar{a}_{S} \bar{a}_{R}\right]
$$

It formally looks like standard coulomb electron-electron interaction term in (24). With respect to the fact that after application of the Wick's theorem (25) the renormalized Fermi vacuum $\Phi_{0}$ has been introduced and zero-particle (scalar) quantity (27) represents electronic energy of the ground state $\Phi_{0}$ that accounts also for coulomb electron-electron interactions (see (27)) and one-particle term is diagonal (29d) and represents unperturbed HF-orbital energies $\left\{\varepsilon_{P}^{0}\right\}$ of the system (one-electron energy spectrum), then two-particle term (32) represents perturbation part of the electronic Hamiltonian (26). It is related to unbalanced treatment of electrons with parallel and antiparallel spins within one-electron approximations. Since perturbation (32) contains only electron-electron interaction term, contributions of this term represent electron correlation energy of the system in its ground electronic state. In this respect, electron correlation energy is treated as a perturbation. Calculation of the electron correlation energy up to higher order of perturbation theory is usually done by diagrammatic many-body perturbation theory. For correlation energy in second order of perturbation theory, analytic expression for closed-shell system can be derived in a simple form,

$$
E_{\text {corr }}=H_{\left(h_{1} h_{2} p_{1} p_{2}\right)}=\sum_{I J}^{o c c} \sum_{A B}^{\text {unocc }} \frac{\left(2 v_{I J A B}^{0}-v_{I J B A}^{0}\right)}{\left(\varepsilon_{I}^{0}+\varepsilon_{J}^{0}-\varepsilon_{A}^{0}-\varepsilon_{B}^{0}\right)}
$$

From the fact that with respect to Fermi level, for energies of unoccupied states hold $\left\{\varepsilon_{A}^{0}\right\}>$ 0 and for energies of occupied states hold $\left\{\varepsilon_{I}^{0}\right\}<0$, follows that $E_{\text {corr }}$ is negative, i.e. it 
decreases electronic energy of the ground state (this contribution corrects total electronic energy of the system $(30,31 c))$. It holds for arbitrary nuclear geometry $R_{0}$ until system remains in a bound state.

Each eigenfunction $\varphi_{J}$ in Slater determinant (28) can be expressed as a linear combination of the atomic orbital (AO) basis functions $\{\mu\}, \varphi_{P}=\sum_{\mu} c_{\mu_{P}}|\mu\rangle$, that are fixed at the positions of the particular nuclei in frozen nuclear configuration $R_{0}$. It represents fixed basis set $\{\mu(x, 0)\}$.

In second quantization, with single-bar $\bar{a}^{+}$being creation operator of the crude-adiabatic electron, it can be written with respect to Fermi vacuum $|0\rangle$ as,

$\mu(x, 0)=\bar{a}_{\mu}^{+}(x, 0)|0\rangle$

and,

$\varphi_{P}(x, 0)=\sum_{\mu} c_{\mu P}|\mu(x, 0)\rangle=\sum_{\mu} c_{\mu P} \bar{a}_{\mu}^{+}(x, 0)|0\rangle=\bar{a}_{P}^{+}(x, 0)|0\rangle$

For solids, in electronic quasi-momentum $k$-space, the basis functions are Bloch-periodic orbitals $\{\mu(k, x, 0)\}$,

$\mu(k, x, 0)=\frac{1}{\sqrt{N}} \sum_{t R}^{N} e^{i k \cdot t R} \mu(x-t R)$

In (33b), $t R$ is translation vector and $\mu(x-t R)=\mu(x)$. The set of $\{\mu(x)\}$ is fixed basis set $\{\mu(x, 0)\}$ in frozen nuclear configuration $R_{0}(Q=0)$. An eigenfunction $\varphi_{P}$ is then crystal orbital CO $-\varphi_{P}$ (band $\varphi_{P}$ ), which is a linear combination of the Bloch-periodic basis functions $\{\mu(k, x, 0)\}$.

In second quantization it has the form,

$\mu(k, x, 0)=\frac{1}{\sqrt{N}} \sum_{t R}^{N} e^{i k . t R} \bar{a}_{\mu}^{+}(x, 0)|0\rangle$

and,

$$
\begin{aligned}
& \varphi_{P}(k, x, 0)=\sum_{\mu} c_{\mu P}^{(k)}|\mu(k, x, 0)\rangle=\sum_{\mu} c_{\mu P}^{(k)} \frac{1}{\sqrt{N}} \sum_{t R}^{N} e^{i k . t R} \bar{a}_{\mu}^{+}(x, 0)|0\rangle= \\
& =\frac{1}{\sqrt{N}} \sum_{t R}^{N} e^{i k . t R} \bar{a}_{P k}^{+}(k, x, 0)|0\rangle
\end{aligned} .
$$

In this case, occupancy of the band is not distinguished by the index of the band $(P)$ itself but it is determined by the value of $k$-vector of particular band dispersion $\varepsilon_{P}^{0}(k)$ with respect to the energy of Fermi level $\varepsilon_{F}^{0}$.

The electronic Hartree-Fock equations (29b) are solved for different displaced but fixed nuclear configurations $\left\{R_{d}\right\}$ at $R_{e q}$ and potential energy (hyper)surface (PES) can be calculated. It can be done by gradient technique where nuclear force constants are calculated in an analytic way by minimization of the total electronic energy (30) as a function of nuclear coordinates R (see e.g.[37]). Knowledge of the PES enables calculation of the force constants of vibration motion and subsequently it enables to solve nuclear Schrödinger equation,

$\left\{T_{N}+E_{0}^{t e}(R)-E_{0, v}^{T S}(R)\right\} \chi_{0, v}(R)=0$

with nuclear Hamiltonian,

$H_{N}(R)=T_{N}+E_{0}^{t e}(R)$

i.e. to solve the problem of nuclear motion quantization, 


$$
H_{N} \rightarrow H_{Q}=\sum_{r}^{\bmod } \hbar \omega_{r}\left(\bar{b}_{\breve{r}}^{+} \bar{b}_{r}+1 / 2\right)=\sum_{r}^{\bmod } \hbar \omega_{r}\left(n_{r}+1 / 2\right)
$$

with nuclear vibration wave function,

$$
\chi(R)=\prod_{r}^{\bmod } \chi_{r, n}(R)=\prod_{r}^{\bmod } \frac{1}{\sqrt{n_{r} !}}\left(\bar{b}_{r}^{+}\right)^{n_{r}}|0\rangle
$$

Creation and annihilation operators of crude-adiabatic phonon modes are also written as single-bare operators, $\bar{b}_{r}^{+}, \bar{b}_{r}$. The operator of nuclear displacements out of fixed nuclear geometry $R_{0}\left(\mathrm{R} \propto \mathrm{R}_{0}+\mathrm{Q}\right)$ for the crude-adiabatic normal mode $r$, is $\bar{Q}_{r}=\left(\bar{b}_{r}^{+}+\bar{b}_{\breve{r}}\right)$ and corresponding momentum operator is $\bar{P}_{r}=\left(\bar{b}_{r}-\bar{b}_{\breve{r}}^{+}\right)$.

If the influence of the nuclear displacements out of fixed nuclear geometry $R_{0}$ on the electronic ground state wave function and electronic energy is assumed to be negligible (crude-adiabatic BOA, i.e. $Q$-independent), the wave function of the total system is, $\Psi_{0}(r, R)=\chi(R) \Phi_{0}\left(r, R_{0}\right)$

or in terms of nuclear motion,

$\Psi_{0}(r, Q)=\chi(Q) \Phi_{0}(r, 0)$

Energy of the total system in the ground electronic state is,

$$
E_{0}^{T S}\left(R_{0}\right)=E_{0}^{t e}\left(R_{0}\right)+\sum_{r} \hbar \omega_{r}\left(n_{r}+1 / 2\right)
$$

For fermion and boson creation and annihilation operators, the standard anti-commutation and commutation relations hold,

$\left\{\bar{a}_{P}, \bar{a}_{Q}\right\}=0, \quad\left\{\bar{a}_{P}, \bar{a}_{Q}^{+}\right\}=\delta_{P Q}$

$\left[\bar{b}_{r}, \bar{b}_{s}\right]=0, \quad\left[\bar{b}_{r}, \bar{b}_{s}^{+}\right]=\delta_{r s}$

"Independence" of fermions and bosons, i.e. possibility of simultaneous diagonalization of electronic and nuclear part of system Hamiltonian, requires also validity of the following commutation relations,

$\left[\bar{a}_{P}, \bar{b}_{r}\right]=0,\left[\bar{a}_{P}, \bar{b}_{r}^{+}\right]=0$

If it is necessary, the effect of electron-vibration (phonon) coupling on the electronic energy and vibration (phonon) spectra are usually calculated by perturbation theory as the corrections to the crude adiabatic ground electronic state and phonons renormalization. On the crude-BOA level these corrections are neglected, however.

\section{II.2. Adiabatic approximation}

In case the of crude-adiabatic approximation, electrons "see" the nuclei at theirs instantaneous positions at rest and nuclei do not "feel" internal dynamics of electrons. Within the spirit of the BOA it would be correct if the electrons follow nuclear motion instantaneously, i.e. electronic state has to dependent explicitly on instantaneous nuclear positions. In this case, the wave function of the system, instead of the form (36a) with $Q$ independent electronic part should be replaced by $Q$-dependent form, i.e.

$\Psi_{0}(r, Q)=\chi(Q) \Phi_{0}(r, Q)$

For molecular systems an analytic derivative method is used. The nuclear force constants are calculated by diagonalization of the Hartree-Fock equations that are now functions of nuclear coordinates. It results in solution of the Coupled Perturbed Hartree-Fock (CPHF) equations (see e.g. [38,39]).

Alternative treatment to this problem, denoted as quasi-particle transformation technique, has been proposed and elaborated by Svrček [40]. In this treatment, the requirement that electrons follow the nuclear motion adiabatically has been expressed through the fermion and 
boson creation and annihilation operators. In [41] it has been shown that solution of the adiabatic problem by quasi-particle transformation technique is equivalent to the results of the CPHF method. Seemingly it means that there is no extra profit of this treatment. In the present paper it is shown, however, that physical background behind the quasi-particle transformation technique is substantial. It can be effectively generalized that justifies and allows application to more complicated situation when the BOA is not valid, i.e. to study antiadiabatic state $\left|E_{0}^{t e}(R)-E_{n}^{t e}\left(R_{e q}\right)\right|<\hbar \omega_{v}$.

To keep the present paper compact, the main points of the original formulation of the adiabatic quasi-particle transformation technique [40] are presented in Appendix A.

Some aspects of different treatments of electron-vibration (phonon) coupling should also be mentioned. Standard solid-state treatment of electron-phonon interaction is based on perturbation theory. Starting Hamiltonian is,

$$
H=H^{0}+H_{e p} \text {. }
$$

The unperturbed part (to be consistent with crude-adiabatic notation, the single-bare operators are used) has the form,

$$
H^{0}=\sum_{k \sigma} \varepsilon_{k}^{0} \bar{a}_{k \sigma}^{+} \bar{a}_{k \sigma}+\sum_{q} \hbar \omega_{q}\left(\bar{b}_{-q}^{+} \bar{b}_{q}+1 / 2\right)
$$

The perturbation Hamiltonian $H_{e p}$, instead of $\Lambda$-perturbation term $(11,19)$, is represented by an electron-phonon (EP) interaction term. The simplest form of this term is,

$$
H_{e p}=\sum_{k, q, \sigma} u^{q}\left(\bar{b}_{q}+\bar{b}_{-q}^{+}\right) \bar{a}_{k+q, \sigma}^{+} \bar{a}_{k, \sigma}=\sum_{k, q . \sigma} u^{q} \bar{Q}_{q} \bar{a}_{k+q, \sigma}^{+} \bar{a}_{k, \sigma}
$$

It can be derived in the assumption of small perturbation of rigid-periodic lattice potential due to vibration displacements of nuclei out-of equilibrium positions. Within the notation used in the present paper, term (39b) corresponds basically to the first order contribution of Taylor's expansion of the core Hamiltonian $h_{P Q}(Q)$, (see 1, 2), with respect to nuclear displacement $Q$ on the crude-adiabatic level. This is evident from eq. (A19b), when at equilibrium geometry for potential energy of nuclear motion holds $E_{N N}^{r}=0$. The unperturbed Hamiltonian (39a) is represented by the terms (29d) and (34b).

Due to the form of EP interaction $H_{e p}(39 \mathrm{~b})$, the first order perturbation correction to the electronic ground state $\Phi_{0}$, i.e. the diagonal perturbation term equals zero: $\left\langle\Psi_{0}\left|H_{e p}\right| \Psi_{0}\right\rangle=0$. All interesting physics is then related to higher-order contributions with participation of excited electronic states, i.e. the first possible non-zero contributions are in the second order of perturbation theory, i.e. terms of the form $\left\langle\Psi_{0}\left|H_{e p}\right| \Psi_{n}\right\rangle\left\langle\Psi_{n}\left|H_{e p}\right| \Psi_{n}\right\rangle /\left(E_{0}^{T S}-E_{n}^{T S}\right)$. These offdiagonal contributions represent, in this treatment, nonadiabatic corrections to the adiabatic ground state energy $\Delta E_{0}^{t e}$ that is associated with renormalization of the normal mode frequencies $\Delta \omega_{r}$. In solids it is calculated as corrections to dispersion of electronic bands $\Delta \varepsilon_{P}(k)$ and corrections to phonon dispersion $\Delta \omega_{r}(q)$.

Exact treatment of nonadiabatic corrections calculation assumes independent calculation of electronic excited states energies $\left\{E_{n(\neq 0)}^{e}(R)\right\}$, however. It would require new optimization of excited state wave functions $\Phi_{m}(r, R)$. It should be extremely complicated since excited state wave functions have to be orthogonal to the ground state wave function. At practical calculations, an approach is used (see II.1) which is based on the orthonormal orbitals (bands) $\left\{\varphi_{a}\right\}$ already optimized for the ground state electronic wave function $\Phi_{0}\left(r, R_{e q}\right)$. By promotion of electron(s) from occupied orbital(s) $\{\mathrm{I}, \mathrm{J}, .$.$\} to virtual - unoccupied orbital(s)$ 
$\{A, B, \ldots\}$, excited state configurations $\left\{\Phi_{A}\right\}$ can be constructed as a linear combination of corresponding Slater determinants $\left\{\Phi_{I \rightarrow A}\right\}$. It can be shown that, e.g. single-electron excitations yield (for closed-shell system) two excited state electronic configurations - lowest lying excited state that is singly excited triplet state ${ }^{3} \Phi_{A(I \rightarrow A)}$, and singly excited singlet state $^{1} \Phi_{A(I \rightarrow A)}$. Differences in the electronic energies of these excited state configurations with respect to the electronic energy of the ground state are;

${ }^{3} E_{A(I \rightarrow A)}^{e}(R)-E_{0}^{e}(R)=\varepsilon_{A}^{0}(R)-\varepsilon_{I}^{0}(R)-J_{I A}$

for singly excited triplet state and,

${ }^{1} E_{A(I \rightarrow A)}^{e}(R)-E_{0}^{e}(R)=\varepsilon_{A}^{0}(R)-\varepsilon_{I}^{0}(R)-J_{I A}+2 K_{I A}$

for singly excited singlet state.

For approximations that do not consider explicitly for two-electron terms, the differences in energies of singly excited triplet and singlet states with respect to the ground state energy are the same and equal to the difference of the energies of involved orbitals, i.e.

$E_{A(I \rightarrow A)}^{e}(R)-E_{0}^{e}(R)=\varepsilon_{A}^{0}(R)-\varepsilon_{I}^{0}(R)$

Multiple electronic excitations can be calculated in a similar way, by generation of Slater determinants of $p$-particle, $h(\equiv p)$-hole states in notation of particle-hole formalism.

In this way, without an explicit calculation of electronic excited state wave functions, the nonadiabatic corrections to (crude-)adiabatic electronic ground state are calculated through optimized eigenfunctions (i.e. occupied $\left(\varphi_{I}\right)$ and unoccupied - virtual ( $\left.\varphi_{A}\right)$ orbitals) of single Slater determinant of the electronic ground state $\Phi_{0}\left(r, R_{e q}\right)$.

Condition for save application of the BOA, expressed in the terms of the ground state orbital energies, is of the form,

$\left|\varepsilon_{I}^{0}(R)-\varepsilon_{A}^{0}(R)\right|>>\hbar \omega_{r}$

It has to be valid for relevant configuration space $R$ at $R_{e q}$ and for the couple of frontier orbitals, i.e. highest occupied $\varphi_{I} \equiv \varphi_{\text {номо }}$ and lowest unoccupied $\varphi_{A} \equiv \varphi_{\text {LUMO }}$ orbitals. In case of solids, with quasi-continuum of states in complex $k$-space representation this inequality can be rewritten in the form,

$\left|\varepsilon_{S}^{0}\left(k_{c}\right)-\varepsilon_{F}^{0}\right|_{R_{e q}}>>\hbar \omega_{r}$

This relation has to hold over the relevant configuration space $R$ at $R_{e q}$ for energies of all bands (S) of multiband system in analytic critical points $k_{c}$ (ACP - absolute or local maxima, minima and saddle points) of $1^{\text {st }} \mathrm{BZ}$, with respect to the energy of the Fermi level $\varepsilon_{F}^{0}$.

In molecular quantum theory, different treatment of electron-vibration interaction has also been elaborated. It is related to direct calculation of the correction to the ground state total electronic energy $\Delta E_{(a ́ d)}^{0}$ that corrects potential energy of nuclear motion on the adiabatic level. In 1997, Kutzelnigg [42] has proved in a rigorous way that so called Born-Handy ansatz $[43,44]$, is physically correct. The Born-Handy ansatz assumes that the diagonal BornOppenheimer correction (DBOC) to adiabatic electronic state - $\mathrm{B}_{\mathrm{nn}}$ (A10) can be calculated directly in laboratory Cartesian coordinate system. The proof [42] is very crucial result, since it eliminates complicated problem with separation of center-off-mass (COM) motion, which arises at introduction of relative coordinates in a molecule-fixed frame system at practical calculations.

Accordingly, the exact adiabatic correction to the total electronic ground state energy is $B_{00}$ (14), i.e. 


$$
\begin{aligned}
& \Delta E_{(a d)}^{0}\left(R_{0}\right)=\left\langle\Phi_{0}(r, \vec{R})\left|T_{N}\right| \Phi_{0}(r, \vec{R})\right\rangle_{R_{0}}=-\sum_{i} \frac{\hbar^{2}}{2 M_{i}}\left\langle\Phi_{0}(r, \vec{R})\left|\nabla_{i}^{2}\right| \Phi_{0}(r, \vec{R})\right\rangle_{R_{0}}= \\
& =\sum_{i \alpha} \frac{\hbar^{2}}{2 M_{i}}\left\langle\frac{\partial \Phi_{0}(r, \vec{R})}{\partial R_{i \alpha}} \mid \frac{\partial \Phi_{0}(r, \vec{R})}{\partial R_{i \alpha}}\right\rangle_{R_{0}}=\Lambda_{00}=B_{00}
\end{aligned}
$$

The derivatives of the ground state Slater determinant $\Phi_{0}$ in the bracket of (45) are performed with respect to the $R_{i \alpha}$, i.e. with respect to the Cartesian component $\alpha$ of the $i^{\text {th }}$ nucleus.

In 1999 it has been shown [41] that this correction is equal to adiabatic correction (A30a) to the total electronic energy of the ground state $\Phi_{0}$, calculated through the expansion coefficients $C_{P Q}(\bar{Q})$ of the quasi-particle transformation (A2), i.e.

$\Delta E_{(a d)}^{0}\left(R_{0}\right)=\left\langle\Phi_{0}(r, \vec{R})\left|T_{N}\right| \Phi_{0}(r, \vec{R})\right\rangle_{\dot{R}_{0}}=\sum_{r A I} \hbar \omega_{r}\left|c_{A I}^{r}\right|^{2}$

In (46), $c_{A I}^{r}$ stand for the first derivative of expansion coefficients (A2) of the quasi-particle transformation with respect to coordinate $Q_{r}$ of particular normal mode $r$, $c_{A I}^{r}=\frac{\partial c_{A I}}{\partial Q_{r}}$

Within the single Slater determinant representation of the ground electronic state, this relation is exact since the eigenfunctions - spinorbitals $\left\{\varphi_{Q}(R)\right\}$ of the Hartree-Fock equations (29b) are orthonormal and form complete basis set, i.e. closure property holds, $\sum_{Q}\left|\varphi_{Q}(r, R)\right\rangle\left\langle\varphi_{Q}(r, R)\right|=1$

It means that both sub-sets, i.e. occupied $\left\{\varphi_{I}(r, R)\right\}$ and unoccupied $\left\{\varphi_{A}(r, R)\right\}$ orbitals are included at calculation of (46). As it is seen from (46), electronic ground state energy correction is due to virtual transitions between occupied $\left\{\varphi_{I}(r, R)\right\}$ and unoccupied orbitals $\left\{\varphi_{A}(r, R)\right\}$ at nuclear vibration motion. In this respect, even $\Delta E_{(a ́ d)}^{0}$ represents exactly DBOC, it covers basically "nonadiabatic" (off-diagonal) corrections in the sense as these are calculated in second-order of perturbation theory when excited electronic states are approximated through virtual orbitals optimized for electronic ground state $\Phi_{0}\left(r, R_{e q}\right)$ and perturbation is an electron-vibration coupling $H_{e p}$, as it has been discussed above. It can be seen very clearly from the expression for correction to frequency of normal modes (A36a) that is identical to the one derived by perturbation theory (A36b).

Correctness of eq. (45) and (46) has been verified by high precision calculation of $\mathrm{H}_{2}, \mathrm{HD}, \mathrm{D}_{2}$ molecules [41] with respect to the exact results for $\mathrm{H}_{2}$ published by Kolos and Wolniewicz $[45,46]$. This treatment can be used for complex molecular systems and it should be effective also in case of solids.

An important conclusion can be made at this place. Electron-vibration (phonon) interactions on the adiabatic level do not stabilize total electronic energy of the ground state. The adiabatic correction $\Delta E_{(a d)}^{0}$ to the ground state total electronic energy (without correlation energy contribution) is small but always positive, $(45,46)$. More over, correction to the energy of the total system $\Delta E^{T S}$, which is composed of the electronic $\Delta E_{(a d)}^{0}$ correction and corrections $\Delta \omega_{r}$ to vibration (phonon) modes (A36a), is also positive with dominant contribution of the electronic correction, 


$$
\Delta E^{T S}=\sum_{r A I} \hbar \omega_{r}\left(c_{A I}^{r}\right)^{2}\left(1-\frac{2 \hbar \omega_{r}}{\left(\varepsilon_{A}^{0}-\varepsilon_{I}^{0}\right)}\right)
$$

The only possible stabilization contribution to the ground state electronic energy on the adiabatic level can arise from the correction to electron correlation energy (see part II).

This conclusion does not contradict the Jahn-Teller effect, or Peierls distortion in solids. In these cases, decrease of the total electronic energy connected to nuclear displacements from high symmetry to lower symmetry nuclear arrangement appears already on the crudeadiabatic level within the clamped nuclear Hamiltonian approximation and it is related to removing of degeneracy of occupied and unoccupied states (asymmetry in population of degenerate states). Degeneracy that is present at high symmetry nuclear geometry is not present at lower symmetry structure, which in fact represents actual equilibrium nuclear configuration $\mathrm{R}_{\mathrm{eq}}$ with lower total electronic energy as it corresponds to the structure with higher symmetry.

In what follows, a connection of the adiabatic treatment, as presented above and in the Appendix A, to canonical transformations and to introduction of new dynamical variables of the system Hamiltonian is shown. The established link is then extended toward solution of more general problem, when adiabatic condition (43, or 44) is not valid and system is in the intrinsic nonadiabatic (or antiadiabatic) state, i.e. when instead of $(43,44)$ holds $\left|\varepsilon_{I}^{0}(R)-\varepsilon_{A}^{0}(R)\right|<\hbar \omega_{r}$, or $\left|\varepsilon_{S}^{0}\left(k_{c}\right)-\varepsilon_{F}^{0}\right|_{R_{e q}}<\hbar \omega_{r}$ in case of solids.

\section{Base transformation - introduction of new dynamical variables}

\section{III.1. Q-dependent adiabatic transformation}

Adiabatic, nuclear displacement $Q$-dependent electronic wave function $\Phi_{0}(r, Q)$ in (38) assumes existence of complete orthonormal basis set $\left\{\varphi_{R}(x, Q)\right\}$, i.e. validity of the following relations,

$$
\left\langle\varphi_{R}(x, \overline{\bar{Q}}) \varphi_{S}(x, \overline{\bar{Q}})\right\rangle=\delta_{R S}, \sum_{R}\left|\varphi_{R}(x, \overline{\bar{Q}})\right\rangle\left\langle\varphi_{R}(x, \overline{\bar{Q}})\right|=1
$$

Now, electron creation and annihilation operators that correspond to the Q-dependent moving base are denoted as double-bar operators $(\overline{\bar{a}}, \overline{\bar{a}})$. Also the boson operators related to the $Q-$ dependent moving base are written as double-bar operators; $\overline{\bar{Q}}_{r}=\left(\overline{\bar{b}}_{r}^{+}+\overline{\bar{b}}_{\breve{r}}\right), \overline{\bar{P}}_{r}=\left(\begin{array}{c}\overline{\bar{b}}_{r}-\bar{b}_{\breve{r}}^{+} \\ \end{array}\right)$.

Then,

$\left.\left.\overline{\bar{a}}_{R}^{+}(x, \overline{\bar{Q}}) 0\right\rangle=\left|\varphi_{R}(x, \overline{\bar{Q}})\right\rangle, \overline{\bar{a}}_{R}(x, \overline{\bar{Q}}) \varphi_{R}(x, \overline{\bar{Q}})\right\rangle=0$

Since adiabatic electrons remain fermions, the operators have to obey standard fermion anticommutation relations,

$$
\left.\left\{\overline{\bar{a}}_{R}, \bar{a}_{S}^{+}\right\}=\delta_{R S} \quad, \quad \bar{a}_{R}, \bar{a}_{S}\right\}=0
$$

Shorthand notation, $\overline{\bar{a}}_{R} \equiv \overline{\bar{a}}_{R}(x, \overline{\bar{Q}}), \overline{\bar{a}}_{R}^{+} \equiv \overline{\bar{a}}_{R}^{+}(x, \overline{\bar{Q}})$ has been used in (52).

Crude-adiabatic electronic wave function $\Phi_{0}(r, 0)$ that does not depend on the nuclear displacements $Q$ is expanded over fixed basis set functions $\left\{\varphi_{R}(x, 0)\right\}$ that are eigenfunctions of clamped nuclear electronic Hartree-Fock equations (29b). This is complete and orthonormal base,

$$
\left\langle\varphi_{R}(x, 0) \mid \varphi_{S}(x, 0)\right\rangle=\delta_{R S}, \quad \sum_{R}\left|\varphi_{R}(x, 0)\right\rangle\left\langle\varphi_{R}(x, 0)\right|=1
$$


Crude-adiabatic fermion creation and annihilation operators that correspond to the fixed basis set are written as single-bar operators $\left(\bar{a}^{+}, \bar{a}\right)$, i.e.

$\bar{a}_{R}^{+}(x, 0)|0\rangle=\left|\varphi_{R}(x, 0)\right\rangle, \bar{a}_{R}(x, 0)\left|\varphi_{R}(x, 0)\right\rangle=|0\rangle$

Also in this case, the standard anticommutation relations $(37,37 \mathrm{a}, \mathrm{b})$ hold and also in this case shorthand notation has been used, $\bar{a}_{R} \equiv \bar{a}_{R}(x, 0), \bar{a}_{R}^{+} \equiv \bar{a}_{R}^{+}(x, 0)$.

Due to properties $(50,53)$, the two bases are interconnected by the base transformation of the following form,

$\left|\varphi_{R}(x, 0)\right\rangle=\sum_{S}\left|\varphi_{S}(x, \overline{\bar{Q}})\right\rangle\left\langle\varphi_{S}(x, \overline{\bar{Q}}) \mid \varphi_{R}(x, 0)\right\rangle=\sum_{S}\left(c_{R S}(\overline{\bar{Q}})\right)^{+}\left|\varphi_{S}(x, \overline{\bar{Q}})\right\rangle$

Then, for fermion operators of second quantization one can write

$\bar{a}_{R}=\sum_{S} c_{R S}\left(\overline{\bar{Q}} \overline{\bar{a}}_{S}, \bar{a}_{R}^{+}=\sum_{S}\left(c_{R S}(\overline{\bar{Q}})\right)^{+} \bar{a}_{S}^{+}\right.$

Elements $c(Q)$ of the $Q$-dependent transformation matrix $C(Q)$ in $(55,56)$ are,

$c_{R S}(\overline{\bar{Q}})=\left\langle\varphi_{R}(x, 0) \mid \varphi_{S}(x, \overline{\bar{Q}})\right\rangle$

Since,

$c_{R S}^{*}(\overline{\bar{Q}})=\left\langle\varphi_{S}(x, \overline{\bar{Q}}) \mid \varphi_{R}(x, 0)\right\rangle$,

then due to closure property and orthonormality $(50,53)$ of the bases, it can be derived that base transformation matrix $C(Q)$ is an unitary matrix,

$\sum_{T} c_{R T} c_{S T}^{*}=\delta_{R S}=\sum_{T} c_{T R}^{*} c_{T S}, C^{+}=\left(C^{T}\right)^{*}=C^{-1}$

It can be shown that the base transformation is identical with canonical transformation of operators that satisfy anti-commutation relations $(37,52)$.

There are two basic possibilities of canonical transformations;

The standard [47], most frequently used canonical transformation works with the same set of dynamical variables $\left(\mathrm{A}_{v}\right)$, i.e.

$$
\begin{aligned}
& T: H\left(A_{\mu}\right) \equiv \tilde{H}\left(A_{\mu}\right)=H\left(\tilde{A}_{\mu}\right)=H\left(U^{+}\left(A_{v}\right) A_{\mu} U\left(A_{v}\right)\right) \\
& \tilde{A}_{\mu}=U^{+}\left(A_{v}\right) A_{\mu} U\left(A_{v}\right)=f\left(A_{v}\right)
\end{aligned}
$$

Canonical transformation (60) is usually used in an effort to make original Hamiltonian diagonal or "more" diagonal, i.e. to remove off-diagonal interaction matrix elements in a system Hamiltonian. Then, for system Hamiltonian $H=H_{0}+H_{\text {int }}$ at the canonical transformation, the anti-hermitean operator S of unitary matrix $U=\exp (S)$ is "constructed" in the form that eliminates presence of interaction term in transformed Hamiltonian completely or at least up to the first order of commutator expansion, i.e. the condition has to be fulfilled,

$\left[H_{0}, S\right]+H_{\text {int }}=0$

This transformation change the form of the Hamiltonian but preserves original system variables.

The other possibility is that by canonical transformation of operators a set of new dynamical variables $\left(A_{v}^{\prime}\right)$ is introduced,

$A_{\mu} \equiv A_{\mu}\left(A_{v}^{\prime}\right)=U^{+}\left(A_{v}^{\prime}\right) A_{\nu}^{\prime} U\left(A_{v}^{\prime}\right)$

Then Hamiltonian is not transformed itself, it remains of the original form, but its variables $\left(\mathrm{A}_{v}\right)$ are replaced by new variables $\left(A_{v}^{\prime}\right)$,

$H\left(A_{\mu}\right) \equiv H\left(U^{+}\left(A_{v}^{\prime}\right) A_{v}^{\prime} U\left(A_{v}^{\prime}\right)\right)$ 
The Hamiltonian written in new variables is,

$H\left(A_{\mu}\left(A_{v}^{\prime}\right)\right) \equiv \tilde{H}\left(A_{v}^{\prime}\right)$

Since at this transformation, there is not any requirement for fulfillment of condition like (61), the transformation does not make Hamiltonian "more" diagonal, but very often it discloses physical aspects of the problem that are not obvious from non-transformed form of the original variables of system Hamiltonian. Beside the other aspects, appearance of the attractive effective electron-electron interaction term at this transformation will be shown at the other place - part II.

Within this transformation method, for canonical transformation of fermion operators can be written,

$\bar{a}_{R}=U^{+} \overline{\bar{a}}_{R} U, \bar{a}_{R}^{+}=U^{+\bar{a}^{+}} \bar{a}_{R} U$

The unitary matrix $\left(U^{+}=U^{-1}\right)$ is of exponential form $U=e^{S}$ and anti-hermitean operator $\mathrm{S}$ $\left(\mathrm{S}^{+}=-\mathrm{S}\right)$ is of the bilinear form,

$S=\sum_{R S} \gamma_{R S}(\overline{\bar{Q}}) \overline{\bar{a}}_{R}^{+} \overline{\bar{a}}_{S} \quad, \gamma_{R S}^{*}=-\gamma_{S R}$

The $\gamma(\mathrm{Q})$ matrix is Q-dependent. For canonical transformation (65) then holds,

$\left.\left.\bar{a}_{R}=e^{-S} \overline{\bar{a}}_{R} e^{S}=\overline{\bar{a}}_{R}+\frac{1}{1 !}\left[\overline{\bar{a}}_{R}, S\right]+\frac{1}{2 !}\left[\overline{\bar{a}}_{R}, S\right], S\right]+\frac{1}{3 !}\left[\left[\overline{\bar{a}}_{R}, S\right], S\right] S\right]+\ldots \ldots$.

Due to fermion anti-commutation relations (52), the commutation expansion (67) can be summed up in a closed form,

$\bar{a}_{R}=\sum_{S}\left(e^{\gamma_{R S}(\overline{\bar{Q}})}\right) \overline{\bar{a}}_{S}$

In this way, for creation operator can be derived

$\bar{a}_{R}^{+}=\sum_{S}\left(e^{\gamma_{R S}(\overline{\bar{Q}})}\right)^{+} \bar{a}_{S}^{+}$

Comparing $(68,68 a)$ with relations (56), one can see that the base transformation of wave functions is identical with canonical transformation of fermion operators. More over it is identical also with quasi-particle transformation as it is postulated in Appendix A. The exponential form of canonical transformation (67) legitimates also Taylor's expansion of the matrix elements of quasi-particle transformation coefficients $(\mathrm{A} 4,56)$, or what is equivalent, the Taylor's expansion of base transformation coefficients, i.e.

$c_{P Q}(\overline{\bar{Q}})=\sum_{k=0}^{\infty} \frac{1}{k !} \sum_{r_{1} . r_{k}} c_{P Q}^{r_{1}, r_{k}} \overline{\bar{Q}}_{r_{1}} \ldots \overline{\bar{Q}}_{r_{k}}$

The form of transformation relations for boson operators of system Hamiltonian is fully dictated by the factorized form of the total system wave function (38). It expresses possibility of simultaneous - independent diagonalization of electron and boson subsystems. It means that transformed fermion and transformed boson operators obey not only standard anticommutation and commutation relations within the individual subsystems,

$$
\left\{\overline{\bar{a}}_{P}, \overline{\bar{a}}_{Q}\right\}=0,\left\{\begin{array}{c}
\overline{\bar{a}}_{P}, \overline{\bar{a}}_{Q}^{+} \\
\bar{a}
\end{array}=\delta_{\mathrm{PQ}}, \quad \mid \overline{\bar{b}}_{r}, \overline{\bar{b}}_{s}\right\rfloor=0, \quad\left[\overline{\bar{b}}_{r}, \overline{\bar{b}}_{s}^{+}\right]=\delta_{r s},
$$

but transformed operators of both subsystems have to commute mutually like the original operators, i.e. also the following commutation relations have to hold,

$\left[\overline{\bar{a}}_{P}, \overline{\bar{b}}_{r}\right\rfloor=0,\left[\begin{array}{c}\bar{a}_{P}, \overline{\bar{b}}_{r}^{+} \\ r\end{array}\right]=0$ 
With respect to the fermion transformation relations (56), the form of transformation relations for boson operators that fully respects commutation relations (71) is (A3, A10), i.e.

$\bar{b}_{r}=\overline{\overline{b_{r}}}+\sum_{P Q} d_{r P Q}(\overline{\bar{Q}}) \overline{\overline{a_{P}^{+}}} \overline{\overline{a_{Q}}}, \quad \bar{b}_{r}^{+}=\overline{\overline{b_{r}^{+}}}+\sum_{P Q}\left(d_{r P Q}(\overline{\bar{Q}})\right)+\overline{\overline{a_{P}^{+}}} \overline{\overline{a_{Q}}}$

For matrix elements $d(\overline{\bar{Q}})$ of transformation matrix $D(\overline{\bar{Q}})$, the Taylor' expansion is defined,

$d_{r P Q}(\overline{\bar{Q}})=\sum_{k=0}^{\infty} \frac{1}{k !} \sum_{s_{1} . s_{k}} d_{r P Q}^{s_{1} . . s_{k}} \overline{\bar{Q}}_{s_{1}} \ldots \overline{\bar{Q}}_{s_{k}}$

The relation that holds, with respect to (71), between elements $d(\overline{\bar{Q}})$ and $c(\overline{\bar{Q}})$ of transformation matrices is specified in Appendix A - (A6, A7).

It can be shown that adiabatic transformation preserves total number of electrons, and nuclear coordinate operator is invariant under transformation, i.e.

$\overline{\bar{N}}=\sum_{P} \overline{\overline{a_{P}^{+}}} \overline{\overline{a_{P}}}=\sum_{P} \bar{a}_{P}^{+} \bar{a}_{P}=\bar{N}, \quad \overline{\bar{Q}}_{r}=\left(\overline{\bar{b}}_{r}^{+}+\overline{\bar{b}}_{\breve{r}}\right)=\left(\bar{b}_{r}^{+}+\bar{b}_{\breve{r}}\right)={\overline{Q_{r}}}$

Up to first order of Taylor's expansion, the momentum operator $\bar{P}_{r}=\left(\bar{b}_{r}-\bar{b}_{r}^{+}\right)$is transformed as,

$\bar{P}_{r}=\left(\overline{\bar{b}}_{r}-\overline{\bar{b}}_{\breve{r}}^{+}\right)+2 \sum_{P Q} c_{P Q}^{\check{r}^{r}} \overline{\bar{a}}_{P}^{+} \bar{a}_{Q}=\overline{\bar{P}}_{r}+2 \sum_{P Q} c_{P Q} \stackrel{\check{r}}{\bar{a}}_{P}^{+} \overline{\boldsymbol{a}}_{Q}$

The term $\overline{\bar{P}}_{r}=\left(\begin{array}{cc}\bar{b} & -\bar{b}_{\breve{r}}^{+}\end{array}\right)$in (74) is nuclear momentum operator on adiabatic level.

For adiabatic $Q$-dependent spinorbitals $\left|\varphi_{P}(x, \overline{\bar{Q}})\right\rangle$, which are the basis functions of the adiabatic $Q$-dependent electronic wave function of the ground state $\Phi_{0}(r, Q)$, expressed over crude-adiabatic orbitals can be derived;

$\left.\left|\varphi_{P}(x, \overline{\bar{Q}})\right\rangle=\bar{a}_{P}^{+}(x, \overline{\bar{Q}}) 0\right\rangle=\left(\bar{a}_{P}^{+}-\sum_{r R} c_{P R}^{r} \overline{\bar{Q}}_{r} \bar{a}_{R}^{+}+O\left(\overline{\bar{Q}}^{2}\right)\right)|0\rangle=$
$=\left|\varphi_{P}(x, 0)\right\rangle-\sum_{r R} c_{P R}^{r} \overline{\bar{Q}}_{r}\left|\varphi_{R}(x, 0)\right\rangle+\ldots .=\left|\varphi_{P}(x, 0)\right\rangle-\sum_{r R} c_{P R}^{r} \bar{Q}_{r}\left|\varphi_{R}(x, 0)\right\rangle+\ldots$.

As it is seen from (75), adiabatic wave function is modulated by the instantaneous nuclear coordinates $\left\{Q_{r}\right\}$ of particular vibration (phonon) modes $\{r\}$ with the weight proportional to transformation coefficients $c_{P R}^{r}$ (coefficients of transformation matrix in the first order of Taylor's expansion). In Appendix $C$ it is shown that $c_{P R}^{r}$ covers the strength of electronvibration (phonon) coupling up to the first order of Taylor's expansion. From (46) it is seen that these coefficients fully determine also exact adiabatic correction to the electronic energy of the ground state $\Delta E_{(a d)}^{0}$.

\section{III.2. P-dependent nonadiabatic transformation}

Save application of the adiabatic BOA requires the inequality $\left|E_{0}^{t e}(R)-E_{n}^{t e}(R)\right|>>\hbar \omega_{r}$ to hold not only at $R_{e q}$ but also over relevant configuration space $R=R_{e q} \pm \Delta R$ near to $R_{e q}$. Relevant configuration space is represented at least by amplitudes of pertaining vibration (phonon) modes of the system. Let as consider situation when this inequality is valid for $R_{e q}$ but it does not hold for $R=R_{e q} \pm Q$. Within single Slater determinant approximation of the ground electronic state it can be written as,

$\left|\varepsilon_{I}^{0}\left(R_{e q}\right)-\varepsilon_{A}^{0}\left(R_{e q}\right)\right|>>\hbar \omega_{r} \quad Q \rightarrow\left|\varepsilon_{I}^{0}\left(R_{e q} \pm Q\right)-\varepsilon_{A}^{0}\left(R_{e q} \pm Q\right)\right|<\hbar \omega_{r}$ 
Corresponding relation holds for solids,

$\left|\varepsilon_{S}^{0}\left(k_{c}\right)-\varepsilon_{F}^{0}\right|_{R_{e q}}>>\hbar \omega_{r} \quad Q \quad \rightarrow \quad\left|\varepsilon_{S}^{0}\left(k_{c}\right)-\varepsilon_{F}^{0}\right|_{R_{e q} \pm Q}<\hbar \omega_{r}$

The inequalities on the rhs of $(76,77)$ indicate that system at vibration motion is in intrinsic nonadiabatic (or antiadiabatic) state. At these circumstances the BOA in the standard Qdependent form (38) is not valid. The $\Lambda$ term (11) that couples electronic and nuclear motion through nuclear kinetic energy operator can be large and can not be treated as a perturbation. It indicates that at the instantaneous nuclear configuration $\left(R_{e q} \pm Q\right)$, instantaneous nuclear kinetic energy (momenta) has been significantly changed, i.e. $\left\langle T_{N}\right\rangle \rightarrow\left\langle\tilde{T}_{N}\right\rangle$. Electrons at these circumstances are not able to follow nuclear motion adiabatically. It means that electronic wave function, in order to respect this fact, should be dependent not only on instantaneous nuclear coordinates $Q$ but it should also be an explicit function of the instantaneous nuclear momenta $P$, i.e. $\Phi_{0} \equiv \Phi_{0}(r, Q, P)$.

Let us assume that wave function of total system can be found in the following factorized form,

$$
\Psi(r, Q, P)=\sum_{m} \chi_{m}(Q, P) \Phi_{m}(r, Q, P)
$$

The form of the wave function (78) is basically $P$-dependent modification of the original $Q$ dependent BOA - (7).

Like in adiabatic case, solution of the problem will be restricted to electronic ground state, i.e. $\Psi_{0}(r, Q, P)=\chi_{0}(Q, P) \Phi_{0}(r, Q, P)$

It means that effect of nuclear momenta will be covered only in the form of $Q, P$-dependent diagonal correction $\Lambda_{00}(Q, P)=\left\langle\Phi_{0}(r, Q, P)\left|\tilde{T}_{N}\right| \Phi_{0}(r, Q, P)\right\rangle$, i.e. in a similar way as it has been covered the effect of instantaneous nuclear coordinates $Q$ on the adiabatic level (45), i.e. $Q$-dependent adiabatic DBOC, $\Lambda_{00}(\overline{\bar{Q}})=\left\langle\Phi_{0}(r, \overline{\bar{Q}})\left|T_{N}\right| \Phi_{0}(r, \overline{\bar{Q}})\right\rangle$.

Solution of this problem is similar to the transition from crude-adiabatic to adiabatic level, as presented above. Now, however, the transition from adiabatic to antiadiabatic level is established.

Nonadiabatic, nuclear displacement and momentum $(Q, P)$-dependent electronic wave function $\Phi_{0}(r, Q, P)$ in (79) assumes existence of complete orthonormal basis set $\left\{\varphi_{R}(x, Q, P)\right\}$, i.e. validity of the following relations,

$\left\langle\varphi_{R}(x, Q, P) \mid \varphi_{S}(x, Q, P)\right\rangle=\delta_{R S}, \sum_{R}\left|\varphi_{R}(x, Q, P)\right\rangle\left\langle\varphi_{R}(x, Q, P)\right|=1$

Electron creation and annihilation operators that correspond to the $(Q, P)$-dependent moving base are written as bar-less operators $\left(a^{+}, a\right)$. Boson operators related to the $(Q, P)$-dependent moving base are denoted also as bar-less operators, $Q_{r}=\left(b_{r}^{+}+b_{\breve{r}}\right)$ and $P_{r}=\left(b_{r}-b_{r}^{+}\right)$. Then,

$a_{R}^{+}(x, Q, P)|0\rangle=\left|\varphi_{R}(x, Q, P)\right\rangle, a_{R}(x, Q, P)\left|\varphi_{R}(x, Q, P)\right\rangle=|0\rangle$

Since nonadiabatic electrons remain fermions, the operators obey standard fermion anticommutation relations,

$\left\{a_{R}, a_{S}^{+}\right\}=\delta_{R S},\left\{a_{R}, a_{S}\right\}=0$

In (82), shorthand notation is used, $a_{R} \equiv a_{R}(x, Q, P)$ and $a_{R}^{+} \equiv a_{R}^{+}(x, Q, P)$.

Since adiabatic $Q$-dependent moving base derived by adiabatic transformation is complete and orthonormal (50), then due to (80), the base transformation to nonadiabatic $(Q, P)$ dependent moving base can be established over the base transformation relation, 


$$
\left|\varphi_{R}(x, \overline{\bar{Q}})\right\rangle=\sum_{S}\left|\varphi_{S}(x, Q, P)\right\rangle\left\langle\varphi_{S}(x, Q, P) \mid \varphi_{R}(x, \overline{\bar{Q}})\right\rangle=\sum_{S}\left(\hat{c}_{R S}(P)\right)^{+}\left|\varphi_{S}(x, Q, P)\right\rangle
$$

For fermion operators of second quantization follow,

$$
\overline{\bar{a}}_{R}=\sum_{S} \hat{c}_{R S}(P) a_{S}, \quad \overline{\bar{a}}_{R}^{+}=\sum_{S}\left(\hat{c}_{R S}(P)\right)^{+} a_{S}^{+}
$$

Elements of the $P$-dependent transformation matrix $\hat{C}(P)$ are,

$$
\hat{c}_{R S}(P)=\left\langle\varphi_{R}(x, \overline{\bar{Q}}) \mid \varphi_{S}(x, Q, P)\right\rangle, c_{R S}^{*}(P)=\left\langle\varphi_{S}(x, Q, P) \mid \varphi_{R}(x, \overline{\bar{Q}})\right\rangle
$$

The $P$-dependent transformation matrix $\hat{C}(P)$ is also unitary matrix, i.e. the relations hold,

$$
\sum_{T} \hat{c}_{R T} \hat{C}_{S T}^{*}=\delta_{R S}=\sum_{T} \hat{c}_{T R}^{*} \hat{c}_{T S}, \hat{C}^{+}=\left(\hat{C}^{T}\right)^{*}=\hat{C}^{-1}
$$

The form of transformation relations for boson operators of system Hamiltonian is fully dictated now by the factorized form of the total system wave function (79). Also in this case, it expresses possibility of simultaneous, independent diagonalization of electron and boson subsystems. It means that transformed-nonadiabatic fermion and transformed nonadiabatic boson operators obey not only standard anticommutation and commutation relations within the individual subsystems,

$$
\left\{a_{P}, a_{Q}\right\}=0,\left\{a_{P}, a_{Q}^{+}\right\}=\delta_{P Q},\left[b_{r}, b_{s}\right]=0,\left[b_{r}, b_{s}^{+}\right]=\delta_{r s},
$$

but, like the original and adiabatic operators, transformed nonadiabatic operators of both subsystems have to commute mutually, i.e. also commutation relations have to hold,

$$
\left[a_{P}, b_{r}\right]=0,\left[a_{P}, b_{r}^{+}\right]=0
$$

With respect to the fermion transformation relations (84), the form of transformation relations for boson operators that fully respects conditions (87) is,

$\overline{\bar{b}}_{r}=b_{r}+\sum_{P Q} \hat{d}_{r P Q}(P) a_{P}^{+} a_{Q}, \quad \overline{\bar{b}}_{r}^{+}=b_{r}^{+}+\sum_{P Q}\left(\hat{d}_{r P Q}(P)\right)^{+} a_{P}^{+} a_{Q}$

At this moment, the canonical transformation can be realized. The new nonadiabatic, barless operators $(84,88)$, replace adiabatic double-bar operators in the adiabatic form of system Hamiltonian (B20a-20e). More details of this transformation, relation between $\hat{d}(P)$ and $\hat{c}(P)$ elements of transformation matrices and treatment of the resulting nonadiabatic system Hamiltonian is presented in [33a] and in the Appendix B.

It can be shown that also this transformation preserve total number of particles, i.e.

$$
N=\sum_{P} a_{P}^{+} a_{P}=\overline{\bar{N}}=\sum_{P} \overline{\overline{a_{P}^{+}}} \overline{\overline{a_{P}}}=\sum_{P} \bar{a}_{P}^{+} \bar{a}_{P}=\bar{N}
$$

Invariant of transformation is now momentum operator,

$$
P_{r}=\left(b_{r}-b_{\breve{r}}^{+}\right)=\overline{\bar{P}}_{r}=\left(\overline{\bar{b}}_{r}-\overline{\bar{b}}_{\breve{r}}^{+}\right) \neq \bar{P}=\left(\bar{b}_{r}-\bar{b}_{\check{r}}^{+}\right), \quad P_{r}=\overline{\bar{P}}_{r} \neq \bar{P}_{r}
$$

However, coordinate operator is transformed up to first order of Taylor's expansion, as,

$$
\begin{aligned}
& Q_{r}=\left(b_{r}+b_{\breve{r}}^{+}\right)=\left(\overline{\bar{b}}_{r}+\overline{\bar{b}}_{\breve{r}}^{+}\right)-2 \sum_{P Q} \hat{c}_{P Q}^{\check{r}}(P) a_{P}^{+} a_{Q}=\overline{\bar{Q}}_{r}-2 \sum_{P Q} \hat{c}_{P Q}^{\check{r}}(P) a_{P}^{+} a_{Q}= \\
& =\bar{Q}_{r}-2 \sum_{P Q} \hat{c}_{P Q}^{\check{r}}(P) a_{P}^{+} a_{Q}
\end{aligned}
$$

i.e.

$\bar{Q}_{r}=\overline{\bar{Q}}_{r} \neq Q_{r}$ 
For nonadiabatic $(Q, P)$-dependent spinorbitals $\left|\varphi_{P}(x, Q, P)\right\rangle$, which are the basis functions of the nonadiabatic $(Q, P)$-dependent electronic wave function of the ground state $\Phi_{0}(r, Q, P)$, expressed over crude-adiabatic orbitals can be derived;

$$
\begin{aligned}
& \left.\left|\varphi_{P}(x, Q, P)\right\rangle=a_{P}^{+}(x, Q, P)|0\rangle=\left(\bar{a}_{P}^{+}-\sum_{r R} c_{P R}^{r} \bar{Q}_{r} \bar{a}_{R}^{+}-\sum_{\breve{r} R} \widehat{c}_{P R}^{r} P_{\breve{r}} \bar{a}_{R}^{+}+O\left(\bar{Q}^{2}, \bar{Q} P, P^{2}\right)\right) \| 0\right\rangle= \\
& =\left|\varphi_{P}(x, 0,0)\right\rangle-\sum_{r R} c_{P R}^{r} \bar{Q}_{r}\left|\varphi_{R}(x, 0,0)\right\rangle-\sum_{\breve{r} R} \widehat{c}_{P R}^{r} \bar{P}_{\breve{r}}\left|\varphi_{R}(x, 0,0)\right\rangle+\ldots \ldots .
\end{aligned}
$$

Nonadiabatic wave function (92) in contrast to adiabatic wave function (75) is modulated not only by the instantaneous nuclear coordinates $\left\{\bar{Q}_{r}\right\}$ of particular vibration (phonon) modes $\{r\}$ but modulation is also over corresponding instantaneous nuclear momenta $\left\{P_{\breve{r}}\right\}$. The weight of momentum modulation is proportional to the $P$-dependent transformation coefficients $\widehat{C}_{P R}^{r}$. It represents first derivative of $\hat{c}_{P R}$ matrix element with respect to nuclear momentum $P_{r}$ (coefficient of transformation matrix in first order of Taylor's expansion),

$\hat{c}_{P R}^{r}=\frac{\partial \hat{c}_{P R}(P)}{\partial P_{r}}$

From Appendix C, it is obvious that these coefficients reflect not only strength of electronvibration (phonon) coupling but mainly magnitude of nonadiabaticity. For nonadiabatic situation, i.e. antiadiabatic state $\left|\varepsilon_{I}^{0}(R)-\varepsilon_{A}^{0}(R)\right|<\hbar \omega_{r}$, the weight of such $P$-modulated state can be considerably large.

\section{Dependence of electronic energy on nuclear vibration displacements and momenta}

Nuclear Q,P-dependent form of the transformed electronic Hamiltonian (Appendix B) and approximate solution of the coefficients of transformation matrices (Appendix C) allow straightforward derivation of approximate analytic forms of the electronic energy corrections. These corrections are calculated with respect to electronic energy terms that are obtained on crude-adiabatic level at particular fixed nuclear configuration $R_{0}$.

IV.1. Correction to electronic ground state energy - zero-particle term correction

With respect to the solution (C2a,b) of approximate GCPHF equations (C1a,b), correction to the electronic ground state energy, i.e. zero-particle term correction (B10a) is,

$$
\Delta E_{(n a)}^{0}=\sum_{r A I} \hbar \omega_{r}\left(\left|c_{A I}^{r}\right|^{2}-\left|\hat{c}_{A I}^{r}\right|^{2}\right)=\sum_{A}^{\text {unocc occ }} \sum_{I} \sum_{r}\left|u_{A I}^{r}\right|^{2} \frac{\hbar \omega_{r}}{\left(\varepsilon_{A}^{0}-\varepsilon_{I}^{0}\right)^{2}-\left(\hbar \omega_{r}\right)^{2}}=\sum_{A}^{\text {unocc occ }} \sum_{I} \Omega_{A I}
$$

The $\Omega$ matrix is a symmetric matrix of the form,

$$
\Omega_{P Q}=\sum_{r} \hbar \omega_{r}\left(\left|c_{P Q}^{r}\right|^{2}-\left|\hat{c}_{P Q}^{r}\right|^{2}\right)=\sum_{r}\left|u_{P Q}^{r}\right|^{2} \frac{\hbar \omega_{r}}{\left(\varepsilon_{P}^{0}-\varepsilon_{Q}^{0}\right)^{2}-\left(\hbar \omega_{r}\right)^{2}}
$$

As it seen from (93), for standard adiabatic $Q$-dependent state, the electronic ground state energy correction $\Delta E_{(n a)}^{e}$ is reduced to the adiabatic DBOC $\Delta E_{(a d)}^{e}(A 30 a, 46)$ which is always positive. In the extreme case (C3a) of strong adiabatic limit $\hbar \omega_{r} /\left|\varepsilon_{P}^{0}-\varepsilon_{Q}^{0}\right| \rightarrow 0$, the correction is basically zero,

$$
\Delta E_{(\text {sad })}^{0}=\sum_{r A I} \hbar \omega_{r}\left(\left|c_{A I}^{r}\right|^{2}\right)=\sum_{r A I}\left|u_{A I}^{r}\right|^{2} \frac{\hbar \omega_{r}}{\left(\varepsilon_{A}^{0}-\varepsilon_{I}^{0}\right)^{2}} \rightarrow 0
$$

For an antiadiabatic state when inequality $\left|\varepsilon_{I}^{0}(R)-\varepsilon_{A}^{0}(R)\right|<\hbar \omega_{r}$ holds, correction to electronic ground state energy (93) is negative and represents stabilization contribution to the 
electronic ground state energy. This contribution can be considerably large and reach the extreme negative value for left-hand side limit toward singular point in (93). Singular point itself is excluded (Appendix C). The correction is always negative for the extreme case (C3b) of strong nonadiabatic limit, $\hbar \omega_{r} /\left|\varepsilon_{P}^{0}-\varepsilon_{Q}^{0}\right| \rightarrow \infty$. However, the contribution in this case does not represent the largest possible negative value and it is equal to,

$$
\Delta E_{(\text {sna })}^{0}=-\sum_{r A I} \hbar \omega_{r}\left(\left|\hat{c}_{A I}^{r}\right|^{2}\right)=-\sum_{r A I} \frac{\left|u_{A I}^{r}\right|^{2}}{\hbar \omega_{r}}
$$

For quasi-momentum $k, q$-space representation of multiband solids, the corresponding equation for correction to electronic ground state energy can be derived straightforwardly from (93). It is based on the correspondence relations for boson and fermion quantities in real space and complex quasi-momentum space representations. In particular, the following transcription relations are used;

normal modes: $r \rightarrow q, \breve{r} \rightarrow-q$

occupied spinorbital: $I \rightarrow R k, \sigma$ - with $\sigma$ spin and occupation factor $f_{k}$ that obey Fermi-Dirac statistics (for $\mathrm{T}=0 \mathrm{~K}, f_{k}=1$, i.e. occupied state below Fermi level),

unoccupied spinorbital: $A \rightarrow S k^{\prime}, \sigma^{\prime}$ - with occupation factor $\left(1-f_{k^{\prime}}\right)$ that obey Fermi-Dirac statistics (for $\mathrm{T}=0 \mathrm{~K}, f_{k^{\prime}}=0$, i.e. unoccupied state above Fermi level)

one-electron HF-orbital energies: occupied states below Fermi level, $\varepsilon_{I}^{0} \rightarrow \varepsilon_{R k}^{0}$

unoccupied states above Fermi level, $\varepsilon_{A}^{0} \rightarrow \varepsilon_{S k^{\prime}}^{0}$

matrix element of electron-vibration(phonon) coupling with quasi-momentum conservation: $u_{A I}^{r} \rightarrow u_{k^{\prime} k}^{q}=u^{q}=u^{k^{\prime}-k}$

Then, with respect to $(k ;-k)$ symmetry, the temperature dependent form of energy correction to electronic ground state (93) in quasi-momentum space representation has the form,

$$
\Delta E_{(n a)}^{0}=2\left(\sum_{R(k), S\left(k^{\prime}\right)}\left(\sum_{k<k_{F}, k^{\prime}>k_{F}}\left|u^{k^{\prime}-k}\right|^{2} f_{k}\left(1-f_{k^{\prime}}\right) \frac{\hbar \omega_{k^{\prime}-k}}{\left(\varepsilon_{k^{\prime}}^{0}-\varepsilon_{k}^{0}\right)^{2}-\left(\hbar \omega_{k^{\prime}-k}\right)^{2}}\right)\right), \varphi_{R k} \neq \varphi_{S k^{\prime}},
$$

Summations in (94) are over all bands $\left\{\varphi_{R}, \varphi_{S}\right\}$ and $k$ points of $1^{\text {st }} \mathrm{BZ}$ of multiband system, including intraband contributions, i.e. $\varphi_{R k}, \varphi_{R k^{\prime}}, k \neq k^{\prime}$, while $\varepsilon_{k}^{0}<\varepsilon_{F} ; \varepsilon_{k^{\prime}}^{0}>\varepsilon_{F}$.

For $\mathrm{T}=0 \mathrm{~K}$, relation (94) is reduced to,

$$
\Delta E_{(n a)}^{0}=2\left(\sum_{R(k), S\left(k^{\prime}\right)}\left(\sum_{k k^{\prime}}\left|u^{k^{\prime}-k}\right|^{2} \frac{\hbar \omega_{k^{\prime}-k}}{\left(\varepsilon_{k^{\prime}}^{0}-\varepsilon_{k}^{0}\right)^{2}-\left(\hbar \omega_{k^{\prime}-k}\right)^{2}}\right)\right)
$$

In (94a), the wave vector $k$ corresponds to states fully occupied below Fermi level $\left(f_{k}=1\right)$, and wave vector $k^{\prime}$ corresponds to empty - virtual states above Fermi-level $\left(f_{k^{\prime}}=0\right)$.

\section{IV.2. Corrections to one-particle term}

Nonadiabatic form of one-particle pure fermion part of the Hamiltonian (boson excitations independent) has the form (B11). There is also boson excitations dependent part, which is represented in Appendix B by the expression valid for boson vacuum (B14b). The $Q, P$-dependent corrections are represented by terms that follow after the first crude-adiabatic term in (B11) and by all terms of (B14b). Restriction to first order of electronvibration(phonon) coupling allows to neglect first summation in (B14b) and for equilibrium geometry the second term in (B11) equals zero. The terms that are the products of electron- 
vibration(phonon) coupling and coulomb two-electron interactions can be expected to be negligible comparing to electron-vibration(phonon) coupling terms and can also be neglected (fifth sum in B11 and third and forth sum in B14b). In solids, due to translation symmetry, the forth sum in (B11) equals zero. Then, one-particle correction has the form,

$$
\begin{aligned}
& \Delta H_{e p}^{\prime}=\sum_{r P Q} \hbar \omega_{r}\left(\sum_{A}\left(c_{P A}^{r} c_{Q A}^{r^{*}}-\hat{c}_{P A}^{r} \hat{c}_{Q A}^{r^{*}}\right)-\sum_{I}\left(c_{P I}^{r} c_{Q I}^{r^{*}}-\hat{c}_{P I}^{r} \hat{c}_{Q I}^{r^{*}}\right)\right) N\left[a_{P}^{+} a_{Q}\right]+ \\
& +\sum_{r P R}\left(\left(\varepsilon_{P}^{0}-\varepsilon_{R}^{0}\right)\left(\left|c_{P R}^{r}\right|^{2}+\left|\hat{c}_{P R}^{r}\right|^{2}\right)-2 \hbar \omega_{r} \operatorname{Re}\left(\hat{c}_{P R}^{r} c_{P R}^{r^{*}}\right)\right) N\left[a_{P}^{+} a_{P}\right]
\end{aligned}
$$

\section{IV.2.1. Nonadiabatic polarons}

The diagonal form of the one-particle correction (95) is,

$$
\begin{aligned}
& \Delta H_{e p}^{\prime}(d g)=\sum_{r P} \hbar \omega_{r}\left(\sum_{A}\left(\left|c_{P A}^{r}\right|^{2}-\left|\hat{c}_{P A}^{r}\right|^{2}\right)-\sum_{I}\left(\left|c_{P I}^{r}\right|^{2}-\left|\hat{c}_{P I}^{r}\right|^{2}\right)\right) N\left[a_{P}^{+} a_{P}\right]+ \\
& +\sum_{r P R}\left(\left(\varepsilon_{P}^{0}-\varepsilon_{R}^{0}\right)\left(\left|c_{P R}^{r}\right|^{2}+\left|\hat{c}_{P R}^{r}\right|^{2}\right)-2 \hbar \omega_{r} \operatorname{Re}\left(\hat{c}_{P R}^{r} c_{P R}^{r^{*}}\right)\right) N\left[a_{P}^{+} a_{P}\right]
\end{aligned}
$$

Substitution for transformation coefficients (C2a,b) yields simple expression for electronvibration(phonon) interaction part of the Hamiltonian,

$$
\Delta H_{e p}^{\prime}(d g)=\sum_{r P}\left(\sum_{A \neq P} \frac{\left|u_{P A}^{r}\right|^{2}}{\varepsilon_{P}^{0}-\varepsilon_{A}^{0}-\hbar \omega_{r}}+\sum_{I \neq P} \frac{\left|u_{P I}^{r}\right|^{2}}{\varepsilon_{P}^{0}-\varepsilon_{I}^{0}+\hbar \omega_{r}}\right) N\left[a_{P}^{+} a_{P}\right]
$$

It can be rearranged into the form that is more convenient for solid state interpretation,

$$
\begin{aligned}
& \Delta H_{e p}^{\prime}(d g)=\sum_{r P R(P \neq R)}\left|u_{P R}^{r}\right|^{2} \frac{1}{\left(\varepsilon_{P}^{0}-\varepsilon_{R}^{0}\right)-\hbar \omega_{r}} N\left[a_{P}^{+} a_{P}\right]- \\
& -2 \sum_{r P I(P \neq I)}\left|u_{P I}^{r}\right|^{2} \frac{\hbar \omega_{r}}{\left(\varepsilon_{P}^{0}-\varepsilon_{I}^{0}\right)^{2}-\left(\hbar \omega_{r}\right)^{2}} N\left[a_{P}^{+} a_{P}\right]
\end{aligned}
$$

Transcription of (96b) to quasi-momentum $k, q$-space of multiband solids is based on the following correspondence: $r \rightarrow q ; P \rightarrow P k, \sigma ; R \rightarrow R k-q, \sigma ; I \rightarrow S k-q, \sigma$ (occupation factor $\left.f_{k-q}\right) ; \varepsilon_{P}^{0} \rightarrow \varepsilon_{k}^{0} ; \varepsilon_{R}^{0} \rightarrow \varepsilon_{k-q}^{0} ; \varepsilon_{I}^{0} \rightarrow \varepsilon_{k-q}^{0}$. The resulting form is,

$$
\begin{aligned}
& \Delta H_{e p}^{\prime}(d g)=\sum_{P k, R k-q}\left(\sum_{q k \sigma}\left|u^{q}\right|^{2} \frac{1}{\left(\varepsilon_{k}^{0}-\varepsilon_{k-q}^{0}\right)-\hbar \omega_{q}} N\left[a_{k, \sigma}^{+} a_{k, \sigma}\right]\right)- \\
& -2 \sum_{P k, S k-q}\left(\sum_{q k \sigma}\left|u^{q}\right|^{2} f_{k-q} \frac{\hbar \omega_{q}}{\left(\varepsilon_{k}^{0}-\varepsilon_{k-q}^{0}\right)^{2}-\left(\hbar \omega_{q}\right)^{2}} N\left[a_{k, \sigma}^{+} a_{k, \sigma}\right]\right)
\end{aligned}
$$

Expression (96c) represents total one-electron energy correction on the general $Q, P$ dependent level due to electron-vibration(phonon) interactions. The first term of (96c) is standard adiabatic (Q-dependent) polaron as it can be derived from Fröhlich Hamiltonian by the Lee-Low-Pines transformation [48]. The second term of (96c) is the correction to polaron energy that arises due to dependence of electronic motion not only on nuclear coordinates but also on the nuclear momenta $P$ (nonadiabatic modification of the BOA). This term can be interpreted as a correction to the energy of individual polaron by an effective field created by all other polarons of the system. 
IV.2.2. Correction to orbital energies of occupied and unoccupied states. Energy gap opening in one-particle spectrum of quasi-degenerate states at Fermi level

The expression (96) for correction to one-particle term covers contributions of boson excitation independent part (first sum) as well of boson excitations dependent part (second sum). At finite temperature, due to boson excitations, contribution of the second term in (96) will be reproduced as multiples of this term, which by itself represents contribution at boson vacuum $(0 \mathrm{~K})$. Since second sum runs over all states, occupied and unoccupied, this contribution does not change character of one-electron spectrum (i.e. position of energy levels with respect to Fermi level), only population of states is changed. More over, for quasicontinuum of states at Fermi level contribution of this term can be negligibly small since the term is odd function of $\left(\varepsilon_{P}^{0}-\varepsilon_{R}^{0}\right)$ and contributions from occupied and unoccupied states will tend to cancel mutually. On the other hand, character of one-particle spectrum can be changed through contribution of the first term due to separate summations that run separately over occupied and unoccupied states. More-over, magnitude of the change in orbital positions is temperature dependent through the temperature dependence of the population of states.

At these circumstances, for investigation of possible changes in the character of oneelectron spectrum of system due to electron-vibration(phonon) interactions on $Q, P$-dependent level, the first term of (96) is crucial. For correction to orbital energy $\Delta \varepsilon_{P}$ of particular state $\varepsilon_{P}^{0}$ follows,

$$
\Delta \varepsilon_{P}=\sum_{r} \hbar \omega_{r}\left(\sum_{A}\left(\left|c_{P A}^{r}\right|^{2}-\left|\hat{c}_{P A}^{r}\right|^{2}\right)-\sum_{I}\left(\left|c_{P I}^{r}\right|^{2}-\left|\hat{c}_{P I}^{r}\right|^{2}\right)\right)=\left(\sum_{A}^{\text {unocc }} \Omega_{P A}-\sum_{I}^{\text {occ }} \Omega_{P I}\right)
$$

Final, corrected orbital energy is,

$$
\varepsilon_{P}=\varepsilon_{P}^{0}+\Delta \varepsilon_{P}
$$

Let us consider only the couple of quasi-degenerate states at Fermi level, occupied state $\varepsilon_{I}^{0}$ and unoccupied state $\varepsilon_{A}^{0}$, a situation that can characterize couple of states in antiadiabatic state, $\left|\varepsilon_{A}^{0}(R)-\varepsilon_{I}^{0}(R)\right|<<\hbar \omega_{r}$. From (97) and from the structure of $\Omega$ matrix (93a) follows directly,

$\Delta \varepsilon_{A}=\left(\varepsilon_{A}-\varepsilon_{A}^{0}\right)=-\Omega_{A I}>0, \Delta \varepsilon_{I}=\Omega_{I A}=\Omega_{I A}<0, \Delta \varepsilon_{I}=-\Delta \varepsilon_{A}$

It means that orbital energy of unoccupied state has been increased, $\varepsilon_{A}>\varepsilon_{A}^{0}$, and orbital energy of the occupied state has been decreased, $\varepsilon_{I}<\varepsilon_{I}^{0}$. The same results follow also from (96a). It confirms the dominant role of the first term in (96) for one-particle spectrum correction. This analysis can be generalized for a set of quasi-degenerate occupied $\{J\}$ and unoccupied $\{B\}$ states (quasi-continuum of states) at Fermi level. Then, with respect to the fact that for antiadiabatic state correction to the ground electronic state (93) is negative $\sum_{A I} \Omega_{A I}<0$, the following relations can be derived,

$$
\Delta \varepsilon_{B}=\sum_{A}^{\text {unocc }} \Omega_{B A}-\sum_{I}^{o c c} \Omega_{B I} \geq 0, \Delta \varepsilon_{J}=\sum_{A}^{\text {unocc }} \Omega_{J A}-\sum_{I}^{o c c} \Omega_{J I} \leq 0,
$$

In particular,

$$
\begin{aligned}
\Delta \varepsilon_{B} & =\sum_{r} \sum_{A}^{\text {unocc }}\left|u_{B A}^{r}\right|^{2} \frac{\hbar \omega_{r}}{\left(\varepsilon_{B}^{0}-\varepsilon_{A}^{0}\right)^{2}-\left(\hbar \omega_{r}\right)^{2}}-\sum_{r} \sum_{I}^{o c c}\left|u_{B I}^{r}\right|^{2} \frac{\hbar \omega_{r}}{\left(\varepsilon_{B}^{0}-\varepsilon_{I}^{0}\right)^{2}-\left(\hbar \omega_{r}\right)^{2}} \\
\Delta \varepsilon_{J} & =\sum_{r} \sum_{A}^{\text {unocc }}\left|u_{J A}^{r}\right|^{2} \frac{\hbar \omega_{r}}{\left(\varepsilon_{J}^{0}-\varepsilon_{A}^{0}\right)^{2}-\left(\hbar \omega_{r}\right)^{2}}-\sum_{r} \sum_{I}^{o c c}\left|u_{J I}^{r}\right|^{2} \frac{\hbar \omega_{r}}{\left(\varepsilon_{J}^{0}-\varepsilon_{I}^{0}\right)^{2}-\left(\hbar \omega_{r}\right)^{2}}
\end{aligned}
$$


At finite temperature $\mathrm{T}$, for a correction $\Delta \varepsilon_{P}$ to an arbitrary state $\varepsilon_{P}^{0}$, from the set of quasidegenerate occupied and unoccupied states at Fermi level can be written, $\Delta \varepsilon_{P}(T)=\sum_{Q} \Omega_{P Q}\left(1-2 f_{Q}\right),\{Q\}$ - set of quasi-degenerate states at Fermi level

The occupation factor $f_{Q}$ obeys Fermi-Dirac statistics,

$$
f_{Q}=\left(\exp \left(\frac{\varepsilon_{Q}-\mu}{k_{B} T}\right)+1\right)^{-1}, \varepsilon_{Q}=\varepsilon_{Q}^{0}+\Delta \varepsilon_{Q}
$$

It is evident that for temperature $0 \mathrm{~K}$, expression (99) reduces to (97).

From (99), temperature dependence of energy gap that is open in one-particle spectrum at Fermi level can be derived [33b] in the form,

$$
\Delta(T)=\Delta(0) \operatorname{tgh}\left(\frac{\Delta(T)}{4 k_{B} T}\right)
$$

The gap is defined as the energy difference of lowest lying corrected unoccupied state $\varepsilon_{B(L U M O)}$ and highest lying corrected occupied state $\varepsilon_{J(\text { номо })}$. At temperature $0 \mathrm{~K}$ holds,

$$
\Delta(0)=\left(\varepsilon_{B(\text { LUMо })}+\left|\varepsilon_{J(\text { номо })}\right|\right)
$$

Factor 4 in the denominator of the argument of $\operatorname{tgh}$ in (100) follows from the assumption that at Fermi level density of quasi-degenerate occupied $\left\{\varepsilon_{J}^{0}\right\}$ and unoccupied $\left\{\varepsilon_{B}^{0}\right\}$ states of the band with gap opening is the same and consequently $\varepsilon_{B(\text { LUMO })}=\left|\varepsilon_{J(\text { номо })}\right|$. This factor can be larger or smaller than 4 , depending on the actual difference in the density of states.

It is trivial to show that the corrections to orbital energies are negligibly small (basically zero) for a system in adiabatic state when $\Delta \varepsilon^{0}>>$.

IV.3. Two-particle term correction. Correction to electron correlation energy

Nonadiabatic form of two-particle pure fermion part of the Hamiltonian (boson excitations independent) has the form (B12). Boson excitations dependent part is represented in the Appendix B by the expression valid for boson vacuum (B14c). The Q,P-dependent corrections are represented by terms that follow in (B12) after the first crude-adiabatic term (electron correlation energy on crude-adiabatic level for fixed nuclear configuration $\mathrm{R}_{0}$ - see 32, 32a) and by all terms of (B14c). Like in the case of treatment of the one-particle term correction, the terms that are the products of electron-vibration(phonon) coupling and coulomb two-electron interactions can be expected to be negligible comparing to electronvibration(phonon) coupling terms, and can be neglected (i.e. all terms in B14c and fourth, fifth, sixth and seventh sum in B12). In solids, due to translation symmetry, the third sum in (B12) equals zero. Then the correction to electron correlation energy due to dependence of electronic motion on nuclear vibration displacements and momenta reduces to a single term,

$$
\Delta H_{e p}^{\prime \prime}=\sum_{r P Q R S} \hbar \omega_{r}\left(c_{P R}^{r} c_{S Q}^{r^{*}}-\hat{c}_{P R}^{r} \hat{c}_{S Q}^{r^{*}}\right) N\left[a_{P}^{+} a_{Q}^{+} a_{S} a_{R}\right]
$$

Substitution for transformation coefficients (C2a,b) yields,

$$
\Delta H_{e p}^{\prime \prime}=\sum_{r P Q R S(P \neq R, Q \neq S)} u_{P R}^{r} u_{S Q}^{r *} \frac{\hbar \omega_{r}\left(\left(\varepsilon_{P}^{0}-\varepsilon_{R}^{0}\right)\left(\varepsilon_{S}^{0}-\varepsilon_{Q}^{0}\right)-\left(\hbar \omega_{r}\right)^{2}\right)}{\left(\left(\varepsilon_{P}^{0}-\varepsilon_{R}^{0}\right)^{2}-\left(\hbar \omega_{r}\right)^{2}\right)\left(\left(\varepsilon_{S}^{0}-\varepsilon_{Q}^{0}\right)^{2}-\left(\hbar \omega_{r}\right)^{2}\right.} N\left[a_{P}^{+} a_{Q}^{+} a_{S} a_{R}\right]
$$

Transcription of (101a) to quasi-momentum $k, q$-space representation of solids is based on the following correspondence: $r \rightarrow q ; P \rightarrow k+q, \sigma ; Q \rightarrow k^{\prime}, \sigma^{\prime} ; R \rightarrow k, \sigma ; S \rightarrow k^{\prime}+q, \sigma^{\prime}$. Resulting final form is, 


$$
\Delta H_{e p}^{\prime \prime}=\sum_{R(k) S\left(k^{\prime}\right)} \sum_{k k^{\prime} q \sigma \sigma^{\prime}(q \neq 0)}\left|u^{q}\right|^{2} \frac{\hbar \omega_{q}\left(\left(\varepsilon_{k+q}^{0}-\varepsilon_{k}^{0}\right)\left(\varepsilon_{k^{\prime}+q}^{0}-\varepsilon_{k^{\prime}}^{0}\right)-\left(\hbar \omega_{q}\right)^{2}\right)}{\left(\left(\varepsilon_{k+q}^{0}-\varepsilon_{k}^{0}\right)^{2}-\left(\hbar \omega_{q}\right)^{2}\right)\left(\left(\varepsilon_{k^{\prime}+q}^{0}-\varepsilon_{k^{\prime}}^{0}\right)^{2}-\left(\hbar \omega_{q}\right)^{2}\right.} N\left[a_{k+q, \sigma}^{+} a_{k^{\prime}, \sigma^{\prime}}^{+} a_{k^{\prime}+q, \sigma^{\prime}} a_{k, \sigma}\right]
$$

\section{Conclusion}

It has been shown that $Q, P$-dependent modification of the BOA for ground electronic state can be derived by sequence of canonical transformations of the basis set functions. The effect of nuclear coordinates $Q$ and momenta $P$ on electronic structure is presented in the form of corrections to zero, one and two-particle terms of clumped nuclear Hamiltonian.

The nuclear dynamics dependence ( $P$-dependent modification of the BOA) is crucial for a system that in the ground electronic state undergoes transition from adiabatic $\left(\omega<<\mathrm{E}_{\mathrm{F}}\right)$ into antiadiabatic $(\omega>$ $\left.E_{F}\right)$ state at vibration motion of nuclei. In the antiadiabatic state, correction to electronic ground state energy (zero-particle correction) is negative and system can be stabilized in antiadiabatic state at distorted geometry with respect to the adiabatic equilibrium structure. Stabilization effect is solely the consequence of nuclear dynamics $(P)$, which is crucial in antiadiabatic state.

One-particle term correction represents correction to polaron energy and corrects also one-particle spectrum of the system. In antiadiabatic state, the gap in one-particle spectrum of quasi-degenerate states (quasi-continuum) at Fermi level can be opened.

Two-particle term correction represents correction to electron correlation energy. It has been shown that nuclear dynamics always increases electron correlation until system, at nuclear motion, remains in a bound state.

In case when system remains at vibration motion of nuclei in adiabatic state, the influence of nuclear dynamics ( $P$-dependence) is negligible. In this case, all basic effects are covered through nuclear coordinates ( $Q$-dependence) within the adiabatic BOA and standard results of solid state (or molecular) physics are recovered.

Corresponding corrections to electronic wave function are also specified. It has been shown that electronic wave function in the antiadiabatic state is strongly modulated by momenta $P$ of nuclei in the phonon mode that induces transition from adiabatic into antiadiabatic state.

Study of the electronic ground state of superconductors from the stand-point of $Q, P$ dependent modification of the BOA is the subject of the following paper - part II.

\section{Acknowledgements}

The author acknowledges financial support of the S-Tech Co., as well as partial support of the grant VEGA1/2465/05 and VEGA1/0013/08.

Appendix A: Adiabatic quasi-particle transformation

In the general form of the electron-vibration Hamiltonian,

$$
H=T_{N}(\bar{P})+E_{N N}(\bar{Q})+\sum_{P Q} h_{P Q}(\bar{Q}) \bar{a}_{P}^{+} \bar{a}_{Q}+\frac{1}{2} \sum_{P Q R S} v_{P Q R S}^{0} \bar{a}_{P}^{+} \bar{a}_{Q}^{+} \bar{a}_{S} \bar{a}_{R},
$$

the crude-adiabatic fermion $\left(\bar{a}^{+}, \bar{a}\right)$ and boson $\left(\bar{b}^{+}, \bar{b}\right)$ creation and annihilation operators are replaced by the new quasi-particle operators, adiabatic fermion and boson creation and annihilation operators $-\left(\overline{\bar{a}^{+}, \overline{\bar{a}}}\right),\left(\overline{\bar{b}}^{+}, \overline{\bar{b}}\right)$,

$$
\begin{aligned}
& \overline{\overline{a_{P}}}=\sum_{Q} c_{P Q}(\bar{Q}) \cdot \bar{a}_{Q}, \quad \overline{\overline{a_{P}^{+}}}=\sum_{Q}\left(c_{P Q}(\bar{Q})\right)^{+} \cdot \bar{a}_{Q}^{+} \\
& \overline{\overline{b_{r}}}=\bar{b}_{r}+\sum_{P Q} d_{r P Q}(\bar{Q}) \bar{a}_{P}^{+} \bar{a}_{Q}, \overline{\overline{b_{r}^{+}}}=\bar{b}_{r}^{+}+\sum_{P Q}\left(d_{r P Q}(\bar{Q})\right)^{+} \bar{a}_{P}^{+} \bar{a}_{Q}
\end{aligned}
$$


The expansion coefficients $c_{P Q}, d_{r P Q}-$ i.e. operators of the quasi-particle transformation (A2, A3), are defined as functions of the nuclear coordinate operators $\bar{Q}$ over the Taylor' series,

$c_{P Q}(\bar{Q})=\sum_{k=0}^{\infty} \frac{1}{k !} \sum_{r_{1} . r_{k}} c_{P Q}^{r_{1} . . r_{k}} \bar{Q}_{r_{1}} \ldots \bar{Q}_{r_{k}}, \quad d_{r P Q}(\bar{Q})=\sum_{k=0}^{\infty} \frac{1}{k !} \sum_{s_{1} . . s_{k}} d_{r P Q}^{s_{1} . . s_{k}} \bar{Q}_{s_{1}} \ldots \bar{Q}_{s_{k}}$

Requirement that the new quasi-particles, $\left(\bar{a}^{+}, \bar{a}\right),\left(\bar{b}^{+}, \bar{b}\right)$, have to obey the same anticommutation an commutation relations as the original crude-adiabatic electrons and phonons (37, 37a,b), results in condition of unitarity for transformation operators $c_{P Q}(\bar{Q})$,

$\sum_{R} c_{P R} C_{Q R}^{+}=\delta_{P Q}$

and in the following relation between $c_{P Q}(\bar{Q})$ and $d_{r P Q}(\bar{Q})$ transformation operators, $d_{r P Q}=\sum_{R} c_{R P}^{+}\left[\bar{b}_{r}, c_{R Q}\right]$ and $d_{r P Q}+d_{\breve{r} Q P}^{+}=0$

Expansion of (A4) up to the second order, which fully cover harmonic vibration, generates the other important relations,

$$
\begin{aligned}
& c_{P Q}^{0}=\delta_{P Q}, c_{P Q}^{r}+c_{Q P}^{\check{r}^{*}}=0, c_{P Q}^{r s}+c_{Q P}^{\breve{r s}^{*}}=-\sum_{R}\left(c_{P R}^{r} c_{Q R}^{\breve{s}^{*}}+c_{P R}^{s} c_{Q R}^{\check{r}^{*}}\right) \\
& d_{r P Q}^{0}=c_{P Q}^{\breve{r}}, d_{r P Q}^{s}=c_{P Q}^{\breve{r} s}+\sum_{R} c_{R P}^{\breve{s}^{*}} c_{R Q}^{\breve{r}}
\end{aligned}
$$

It can be shown that transformations (A2, A3) preserve the total number of fermions and nuclear coordinate operator is invariant of transformation,

$\overline{\bar{N}}=\sum_{P} \overline{\overline{a_{P}^{+}}} \overline{\overline{a_{P}}}=\sum_{P} \bar{a}_{P}^{+} \bar{a}_{P}=\bar{N}, \quad \overline{\overline{Q_{r}}}=\overline{Q_{r}}$

The transformations (A2, A3) form a transformation group, i.e. for inverse transformations hold,

$$
\begin{aligned}
& \bar{a}_{P}=\sum_{Q} c_{P Q}(\overline{\bar{Q}}) \overline{\bar{a}}_{Q}, \quad \bar{a}_{P}^{+}=\sum_{Q}\left(c_{P Q}(\overline{\bar{Q}})\right)^{+} \overline{\overline{. a_{Q}^{+}}} \\
& \bar{b}_{r}=\overline{\overline{b_{r}}}+\sum_{P Q} d_{r P Q}\left(\overline{\bar{Q}} \overline{\overline{a_{P}^{+}}} \overline{\overline{a_{Q}}}, \quad \bar{b}_{r}^{+}=\overline{\overline{b_{r}^{+}}}+\sum_{P Q}\left(d_{r P Q}(\overline{\bar{Q}})\right)^{+} \overline{\overline{a_{P}^{+}}} \overline{\overline{a_{Q}}}\right.
\end{aligned}
$$

whereas,

$$
\begin{aligned}
& c_{P Q}(\overline{\bar{Q}})=c_{P Q}(\bar{Q})=\left(c_{Q P}(\bar{Q})\right)^{+} \\
& d_{r P Q}(\overline{\bar{Q}})=d_{r P Q}(\bar{Q})=-\sum_{R S} c_{P R}(\bar{Q}) d_{r R S}(\bar{Q})\left(c_{Q S}(\bar{Q})\right)^{+}
\end{aligned}
$$

The crucial point at the solution of this problem is quantization of the nuclear part of the Hamiltonian (A1),

$$
H_{N} \rightarrow H_{B}=\sum_{r}^{\bmod } \hbar \omega_{r}\left(\bar{b}_{r}^{+} \bar{b}_{r}+\frac{1}{2}\right)
$$

In general, this part can be written as the sum of nuclear kinetic and nuclear potential energy, $H_{B}=E_{k i n}(\bar{P})+E_{\text {pot }}(\bar{Q})$ whereas, $E_{p o t}(\bar{Q})=E_{N N}^{(2)}(\bar{Q})+V_{N}^{(2)}(\bar{Q})$

The standard, quadratic, part of the nuclear potential energy $E_{N N}^{(2)}(\bar{Q})$ is corrected now by some, yet unknown, potential energy term $V_{N}^{(2)}(\bar{Q})$ that is also quadratic function of coordinate operators. This correction originates from the interaction of vibrating nuclei with 
electrons. In general, kinetic energy of vibration motion can also be corrected by some, yet unknown quadratic function of nuclear momenta operators $W_{N}^{(2)}(\bar{P})$, i.e.

$$
E_{\text {kin }}(\bar{P})=T_{N}(\bar{P})+W_{N}^{(2)}(\bar{P})
$$

On the adiabatic level, however, kinetic energy correction is negligibly small and it is neglected. It becomes important only when the Born-Oppenheimer approximation is broken, $\mid E_{0}^{t e}(R)-E_{n}^{t e}\left(R_{e q}\right) \leq \hbar \omega_{v}$, i.e. in the case when electrons due to theirs final mass are not able to follow nuclear motion adiabatically. This correction is not present in [40] and in what follows it has been introduced just with respect to subsequent nonadiabatic transformation as it is specified in the present work.

The Hamiltonian (A1) can now be formally written as the sum of two parts, electronic $\mathrm{H}_{\mathrm{A}}$ and nuclear part $\mathrm{H}_{\mathrm{B}}$,

$\mathrm{H}=\mathrm{H}_{\mathrm{A}}+\mathrm{H}_{\mathrm{B}}$

The nuclear part is specified by eqs. (A13-16), and $\mathrm{H}_{\mathrm{A}}$ is,

$$
H_{A}=E_{N N}(\bar{Q})-E_{N N}^{(2)}(\bar{Q})-V_{N}^{(2)}(\bar{Q})-W_{N}^{(2)}(\bar{P})+\sum_{P Q} h_{P Q}(\bar{Q}) \bar{a}_{P}^{+} \bar{a}_{Q}+\frac{1}{2} \sum_{P Q R S} v_{P Q R S}^{0} \bar{a}_{P}^{+} \bar{a}_{Q}^{+} \bar{a}_{S} \bar{a}_{R}
$$

For particular orders of the Taylor's expansion, the electronic part (A18) has the form,

$$
\begin{aligned}
& H_{A \overline{0}(0,0)}=E_{N N}^{0}\left(R_{0}\right)+\sum_{P Q} h_{P Q}^{0}\left(R_{0}\right) \bar{a}_{P}^{+} \bar{a}_{Q}+\frac{1}{2} \sum_{P Q R S} v_{P Q R S}^{0} \bar{a}_{P}^{+} \bar{a}_{Q}^{+} \bar{a}_{S} \bar{a}_{R} \\
& H_{A \overline{1}(1,0)}=\sum_{r} E_{N N}^{r} \bar{Q}_{r}+\sum_{R Q r} u_{P Q}^{r} \bar{Q}_{r} \bar{a}_{P}^{+} \bar{a}_{Q} \\
& H_{A \overline{2}(2,0)}=-\frac{1}{2} \sum_{r s} V_{N}^{r s} \bar{Q}_{r} \bar{Q}_{s}+\frac{1}{2} \sum_{P Q r s} u_{P Q}^{r s} \bar{Q}_{r} \bar{Q}_{s} \bar{a}_{P}^{+} \bar{a}_{Q} \\
& H_{A \overline{2}(0,2)}=-\frac{1}{2} \sum_{r s} W_{N}^{r s} \bar{P}_{r} \bar{P}_{s}
\end{aligned}
$$

In the notation used, $H_{A \bar{i}(j, k)}$, $\bar{i}$ stands for the order of Taylor' series expansion in crude adiabatic representation, whereas $j$ stands for the order of the coordinate operator $\bar{Q}$ and $k$ stands for the order of momentum operator $\bar{P}$ within the given order $\bar{i}$ of the Taylor's expansion.

The adiabatic quasi-particle transformations (A2, A3, A9, A10), up to the second order of Taylor's expansion (A4), generate the terms $H_{A \overline{i(j, k)}}$ and $H_{B i \overline{(j, k)}}$ whereas i, j, k in $\overline{\overline{i(j, k)}}$ stand now for the $i$-th order of Taylor' series expansion in adiabatic representation, the $j$-th order of the coordinate operator $\overline{\bar{Q}}$ and the $k$-th order of momentum operator $\overline{\bar{P}}$ within the given order of the Taylor's expansion.

Up to the $2^{\text {nd }}$ order expansion in Q, $\mathrm{P}$ and in $2^{\text {nd }}$ order expansion of the operators of quasiparticle transformation $\left(\bar{c}_{P Q}, \bar{d}_{r P Q}\right)$, the particular terms (A19a-d) and (A13) transform as,

$$
\begin{aligned}
& H_{A \overline{0}(0,0)} \rightarrow H_{\overline{A 0(0,0)}}+H_{\overline{A 1(1,0)}}+H_{\overline{A 2(2,0)}} \\
& H_{A \overline{1}(1,0)} \rightarrow H_{\overline{A 1(1,0)}}+H_{\overline{A 2(2,0)}} \\
& H_{A \overline{2}(2,0)} \rightarrow H_{\overline{A 2(2,0)}} \\
& H_{A \overline{2}(0,2)} \rightarrow H_{\overline{A 2(0,2)}}
\end{aligned}
$$




$$
H_{B}=H_{B \overline{0}(0,0)} \rightarrow H_{\overline{B 0(0,0)}}+H_{\overline{B 1(0,1)}}+H_{\overline{B 2(1,1)}}+H_{\overline{B 2(0,0)}}
$$

The form of transformed terms is,

$$
\begin{aligned}
& H_{\overline{A 0(0,0)}}=E_{N N}^{0}\left(R_{0}\right)+\sum_{P Q} h_{P Q}^{0}\left(R_{0}\right) \bar{a}_{P}^{+} \bar{a}_{Q}+\frac{1}{2} \sum_{P Q R S} v_{P Q R S}^{0} \stackrel{\bar{a}_{P}^{+}=++}{\bar{a}_{Q}} \bar{a}_{S} \overline{\bar{a}}_{R} \\
& H_{\overline{A 1(1,0)}}=\sum_{r} E_{N N}^{r} \overline{\bar{Q}}_{r}+\sum_{P Q r}\left[u_{P Q}^{r}+\sum_{R}\left(h_{P Q}^{0} c_{R Q}^{r}+h_{R Q}^{0} C_{R P}^{\check{r}^{*}}\right)\right] \overline{\bar{Q}}_{r} \bar{a}_{P}^{+} \bar{a}_{Q}+ \\
& +\sum_{P Q R S T r}\left(v_{P Q T S}^{0} c_{T R}^{r}+v_{T Q R S}^{0} c_{T P}^{r^{*}}\right) \overline{\bar{Q}}_{r} \bar{a}_{P}^{+} \bar{a}_{Q}^{+}=\bar{a}_{S} \bar{a}_{R} \\
& H \overline{\overline{A 2(2,0)}}=-\frac{1}{2} \sum_{r s} V_{N}^{r s} \overline{\bar{Q}}_{r} \overline{\bar{Q}}_{s}+ \\
& +\sum_{P Q r s}\left[\frac{1}{2} u_{P Q}^{r s}+\sum_{R}\left(\frac{1}{2} h_{P R}^{0} c_{R Q}^{r s}+\frac{1}{2} h_{R Q}^{0} c_{R P}^{\check{r} \breve{s}^{*}}+u_{P R}^{r} c_{R Q}^{s}+u_{R Q}^{r} c_{R P}^{\breve{s}^{*}}\right)+\sum_{R S} h_{R S}^{0} c_{R P}^{\check{r}^{*}} c_{S Q}^{s}\right] \overline{\bar{Q}} \overline{\bar{Q}}_{s}=++\bar{a}_{P} \bar{a}_{Q}+ \\
& +\frac{1}{2} \sum_{P Q R S T r s}\left\{v_{P Q T S}^{0} c_{T R}^{r s}+v_{T Q R S}^{0} c_{T P}^{\check{r} \breve{s}^{*}}+\sum_{K}\left[v_{P Q T K}^{0} c_{T R}^{r} c_{K S}^{s}+v_{T K R S}^{0} c_{T P}^{\check{r}^{*}} c_{K Q}^{\breve{s}^{*}}+2\left(v_{T Q K S}^{0}-v_{T Q S K}^{0}\right) c_{T P}^{r^{*}} C_{K R}^{s}\right]\right\} . \\
& \overline{\bar{Q}}_{r} \overline{\bar{Q}}_{S} \bar{a}_{P} \bar{a}_{Q} \bar{a}_{S} \bar{a}_{R} \\
& H_{\overline{A 2(0,2)}}=-\frac{1}{2} \sum_{r s} W_{N}^{r s} \overline{\bar{P}}_{r} \overline{\bar{P}}_{s} \\
& H_{\overline{B 0(0,0)}}=H_{B \overline{0}(0,0)}=\sum_{r} \hbar \omega_{r}\left(\overline{\bar{b}}_{r}^{+} \overline{\bar{b}}_{r}+\frac{1}{2}\right) \\
& H_{\overline{B 1(0,1)}}=-\sum_{r P Q} \hbar \omega_{r} d_{\vec{r} P Q}^{0} \overline{\bar{P}}_{r} \bar{a}_{P}^{+} \bar{a}_{Q} \\
& H \overline{B 2(1,1)}=-\frac{1}{2} \sum_{r s P Q} \hbar \omega_{r} d_{r P Q}^{s}\left(\overline{\bar{P}}_{r} \overline{\bar{Q}}_{s}+\overline{\bar{Q}}_{s} \overline{\bar{P}}_{r}\right) \overline{\bar{a}}_{P}^{+} \overline{\bar{a}}_{Q} \\
& H_{\overline{B 2(0,0)}}=-\sum_{P Q R r} \hbar \omega_{r} d_{\breve{r} P R}^{0} d_{r R Q}^{0} \overline{\bar{a}}_{P}^{+} \overline{\bar{a}}_{Q}-\sum_{P Q R S} \hbar \omega_{r} d_{\breve{r} P R}^{0} d_{r Q S}^{0} \bar{a}_{P} \bar{a}_{Q} \bar{a}_{S} \overline{\bar{a}}_{R}
\end{aligned}
$$

Since quasi-particle transformations preserve total number of fermions, the Wick's theorem can be applied to all terms with fermion operators resulting in the normal product form and corresponding contractions. It enables SCF solution of the electron-vibration problem. It should be noticed that after application of Wick's theorem, general set of all spinorbitals (P, $\mathrm{Q}, \mathrm{R}, \mathrm{S}$ ) is divided on two distinct groups, occupied spinorbitals (I,J,..) and unoccupied spinorbitals $(A, B, .$.$) . Treatment of the particular transformed terms and solution of the$ problem is described in [40].

The main points of the solution are:

The pure fermion part of the Hamiltonian (Wick's theorem is applied to all terms with fermion operators, but pure fermion part is represented only by terms without boson operators) is sum of three terms,

$$
H_{F(a d)}\left(R_{0}\right)=H_{F(a d)}^{0}\left(R_{0}\right)+H_{F(a d)}^{\prime}\left(R_{0}\right)+H_{F(a d)}^{\prime \prime}\left(R_{0}\right)
$$

a/ The ground state adiabatic total electronic energy is represented by zero-particle term (i.e. scalar quantity, contribution of contractions of fermion operators), 


$$
H_{F(a d)}^{0}\left(R_{0}\right)=E_{0(c a d)}^{t e}\left(R_{0}\right)+\Delta E_{(a ́ d)}^{0}=\left(E_{N N}^{0}\left(R_{0}\right)+E_{S C F}^{0}\left(R_{0}\right)\right)+\sum_{r A I} \hbar \omega_{r}\left|c_{A I}^{r}\right|^{2}
$$

As it is seen from (A30), the ground state crude-adiabatic total electronic energy $E_{0(\text { cad })}^{\text {te }}\left(R_{0}\right)$ at fixed nuclear geometry $R_{0}$ is due to electron-vibration (phonon) coupling on the adiabatic level corrected by the term,

$\Delta E_{(a ́ d)}^{0}=\sum_{r A I} \hbar \omega_{r}\left|c_{A I}^{r}\right|^{2}$

This correction is contribution of contractions of the one and two-particle terms of (A28). In (A30a), summation runs over all normal modes (r), the unoccupied orbitals (A) and occupied orbitals (I).

b/ The one-particle term covers adiabatic correction to the crude-adiabatic HF orbital energies,

$$
H_{F(a d)}^{\prime}\left(R_{0}\right)=\sum_{P} \varepsilon_{P(c a d)}^{0}\left(R_{0}\right) N\left[\bar{a}_{P}^{+} \bar{a}_{P}\right]+\sum_{r P Q} \hbar \omega_{r}\left(\sum_{A} c_{P A}^{r} c_{Q A}^{r^{*}}-\sum_{I} c_{P I}^{r} c_{Q I}^{r^{*}}\right) N\left[\overline{\bar{a}}_{P}^{+} \bar{a}_{Q}\right]
$$

In (A31), $\{\mathrm{P}, \mathrm{Q}\}$ stands for total set of orbitals, whereas $\{\mathrm{A}\}$ represents the subset of unoccupied orbitals and $\{\mathrm{I}\}$ represents the subset of occupied orbitals.

c/ The two-particle term covers adiabatic correction to the crude-adiabatic electron correlation energy,

$$
H_{F(a d)}^{\prime \prime}\left(R_{0}\right)=\frac{1}{2} \sum_{P Q R S} v_{P Q R S(c a d)}^{0} N\left[\bar{a}_{P}^{+} \bar{a}_{Q}^{+} \bar{a}_{S} \bar{a}_{R}\right]+\sum_{r P Q R S} \hbar \omega_{r}\left(c_{P R}^{r} c_{S Q}^{r^{*}}\right) N\left[\bar{a}_{P} \bar{a}_{Q}^{+} \bar{a}_{S} \bar{a}_{R}\right]
$$

The terms of the Hamiltonian that contain after the transformation and Wick's theorem application the fermion and boson operators, result in the analog of the CPHF equations and in the equation for the nuclear potential energy correction $V_{N}^{r s}$,

$$
u_{P Q}^{r}+\left(\varepsilon_{P}^{0}-\varepsilon_{Q}^{0}\right) c_{P Q}^{r}+\sum_{A I}\left[\left(v_{P I Q A}^{0}-v_{P I A Q}^{0}\right) c_{A I}^{r}-\left(v_{P A Q I}^{0}-v_{I A I Q}^{0}\right) c_{I A}^{r}\right]=\varepsilon_{P}^{r} \delta_{P Q}
$$

and

$V_{N}^{r s}=\sum_{I} u_{I I}^{r s}+\sum_{A I}\left(u_{I A}^{r} c_{A I}^{s}+u_{I A}^{s} c_{A I}^{r}\right)$

Diagonalization of (A33) yields transformation coefficients $\left(c_{P Q}^{r}\right)$. It enables to calculate all corrections on the adiabatic level (see, [40,41]). Knowledge of the nuclear potential energy correction $V_{N}^{r s}$ enables to solve secular equations of nuclear motion,

$$
\sum_{t}\left(\frac{4}{\hbar^{2}} \sum_{s} E_{k i n}^{\check{r s}} E_{p o t}^{\breve{s t}}+\omega_{u}^{2} \delta_{r t}\right) \alpha_{t u}=0
$$

with the normalization condition for eigenvectrors $\alpha$,

$$
\sum_{r s} E_{p o t}^{\check{r s}} \alpha_{r t}^{*} \alpha_{s t}=\frac{\hbar}{2} \omega_{t}
$$

Solution of the electron-vibration problem on the adiabatic level is then an iterative SCF procedure of coupled electronic, $\mathrm{CPHF}$ and nuclear equations. If contribution of the differences in two-electron coulomb terms in (A33), i.e. the third lhs-term in (A33), can be neglected with respect to $\left(\varepsilon_{P}^{0}-\varepsilon_{Q}^{0}\right)$ differences, then formal analytic expression for corrected normal mode frequencies on adiabatic level can be written in the analytic form,

$$
\hbar \omega_{r(a d)}=\hbar \omega_{r}-2\left(\hbar \omega_{r}\right)^{2} \sum_{A I} \frac{1}{\left(\varepsilon_{A}^{0}-\varepsilon_{I}^{0}\right)^{(}\left(c_{A I}^{r}\right)^{2}}
$$

The normal mode energy correction, 


$$
\Delta \omega_{r}=-2\left(\hbar \omega_{r}\right)^{2} \sum_{A I} \frac{1}{\left(\varepsilon_{A}^{0}-\varepsilon_{I}^{0}\right)}\left(c_{A I}^{r}\right)^{2}
$$

is due to adiabatic correction $\left(V_{N}^{r s}\right)$ to nuclear potential energy $E_{N N}^{(2)}$.

The normal mode energy correction (A36a) is identical to the normal mode energy correction that can be obtained as nonadiabatic correction in second order of perturbation theory assuming single boson excitation processes (see expression C2a for $c_{A I}^{r}$ ),

$$
\Delta \omega_{r}=-2\left(\hbar \omega_{r}\right)^{2} \sum_{A I}\left|u_{A I}^{r}\right|^{2} \frac{\left(\varepsilon_{A}^{0}-\varepsilon_{I}^{0}\right)}{\left[\left(\varepsilon_{A}^{0}-\varepsilon_{I}^{0}\right)^{2}-\left(\hbar \omega_{r}\right)^{2}\right]^{2}}
$$

The term nonadiabatic is related to the fact that contributions to the correction arise due to virtual transitions between occupied $\{\mathrm{I}\}$ and unoccupied $\{\mathrm{A}\}$ states. In this case, the unperturbed part of the Hamiltonian is represented by the terms A21, A25 and term A26 represents perturbation.

Appendix B: Nonadiabatic, P-dependent transformation of adiabatic Q-dependent variables. $Q, P$ - dependent form of electronic Hamiltonian

Transformation of adiabatic variables $\left(\bar{a}^{+}, \bar{a}\right),\left(\bar{b}^{+}, \overline{\bar{b}}\right)$ to nonadiabatic variables $\left(a^{+}, a\right),\left(b^{+}, b\right)$ has been introduced in Section III.2. In particular,

$$
\overline{\bar{a}}_{R}=\sum_{S} \hat{c}_{R S}(P) a_{S}, \quad \overline{\bar{a}}_{R}^{+}=\sum_{S}\left(\hat{c}_{R S}(P)\right)^{+} a_{S}^{+}, \quad a_{R}=\sum_{S} \hat{c}_{R S}(\overline{\bar{P}}) \overline{\bar{a}_{S},} a_{R}^{+}=\sum_{S}\left(\hat{c}_{R S}(\overline{\bar{P}})\right)^{+} \overline{\bar{a}}_{S}^{+}
$$

and,

$$
\begin{array}{ll}
\overline{\bar{b}}_{r}=b_{r}+\sum_{P Q} \hat{d}_{r P Q}(P) a_{P}^{+} a_{Q}, & \overline{\bar{b}}_{r}^{+}=b_{r}^{+}+\sum_{P Q}\left(\hat{d}_{r P Q}(P)\right)^{+} a_{P}^{+} a_{Q} \\
b_{r}=\overline{\bar{b}}_{r}+\sum_{P Q} \hat{d}_{r P Q}(\overline{\bar{P}})_{\bar{a}}^{+} \overline{\bar{a}}_{P}, & b_{r}^{+}=\overline{\bar{b}}_{r}^{+}+\sum_{P Q}\left(\hat{d}_{r P Q}(\overline{\bar{P}})\right)^{+} \overline{\bar{a}}_{P}^{+} \overline{\bar{a}}_{Q}
\end{array}
$$

Since transformation matrix $\hat{C}(P)$ is unitary matrix (85a) and anticommutation and commutations relations $(86,87)$ have to hold, then following relation between matrix elements $\hat{c}_{P Q}$ and $\hat{d}_{r P Q}$ of corresponding transformation matrices can be derived,

$\hat{d}_{r P Q}=\sum_{R}\left(\hat{c}_{R P}\right)^{+}\left[\overline{\bar{b}}_{r}, \hat{c}_{R Q}\right\rfloor$

It also holds,

$\hat{d}_{r P Q}-\left(d_{\breve{r} Q P}\right)^{+}=0$

and,

$\hat{c}_{P Q}(P)=\left(\hat{c}_{Q P}(\overline{\bar{P}})\right)^{+}, \hat{d}_{r P Q}(P)=-\sum_{R S} \hat{c}_{P R}(\overline{\bar{P}}) \hat{d}_{r R S}(\overline{\bar{P}})\left(\hat{c}_{Q S}(\overline{\bar{P}})\right)^{+}$

It should be reminded that invariant of the transformation is beside the total number of particles (89) also momentum operator (90), i.e. $P=\overline{\bar{P}}$.

Transformation coefficients $\hat{c}_{P Q}, \hat{d}_{r P Q}$ - i.e. operators of the transformations (B1), are defined as functions of the nuclear momenta operators $\mathrm{P}$ through the Taylor' series,

$\hat{c}_{P Q}(P)=\sum_{k=0}^{\infty} \frac{1}{k !} \sum_{r_{1} \cdot . r_{k}} \hat{c}_{P Q}^{r_{1} . . r_{k}} P_{r_{1}} \ldots P_{r_{k}}, \quad \hat{d}_{r P Q}(P)=\sum_{k=0}^{\infty} \frac{1}{k !} \sum_{s_{1} . . s_{k}} \hat{d}_{r P Q}^{s_{1} . . . s_{k}} P_{s_{1}} \ldots P_{s_{k}}$

Up to second order of Taylor's expansion then holds, 


$$
\begin{aligned}
& \hat{c}_{P Q}^{0}=\delta_{P Q}, \hat{c}_{P Q}^{r}-\hat{c}_{Q P}^{r^{*}}=0, \quad \hat{c}_{P Q}^{r s}+\hat{c}_{Q P}^{\check{r}^{*}}=\sum_{R}\left(\hat{c}_{P R}^{r} \hat{c}_{Q R}^{\breve{s}^{*}}+\hat{c}_{P R}^{s} \hat{c}_{Q R}^{\check{r}^{*}}\right) \\
& \hat{d}_{r P Q}^{0}=-\hat{c}_{P Q}^{\breve{r}}, \hat{d}_{r P Q}^{s}=-\hat{c}_{P Q}^{\check{r}^{s}}+\sum_{R} \hat{c}_{R P}^{\check{s}^{*}} \hat{c}_{R Q}^{\breve{r}}
\end{aligned}
$$

Nonadiabatic, bar-less, variables (B1) are now introduced into the adiabatic form of the Hamiltonian (A20a-A20e) instead of adiabatic double-bar variables. The particular terms, up to second order of Taylor's expansion in boson operators, transform as

$$
\begin{aligned}
& H_{\overline{A 0(0,0)}} \rightarrow H_{A 0(0,0)}+H_{A 1(0,1)}+H_{A 2(0,2)} \\
& H_{\overline{A 1(1,0)}} \rightarrow H_{A 1(1,0)}+H_{A 2(1,1)}+H_{A 2(0,0)} \\
& H_{\overline{A 2(2,0)}} \rightarrow H_{A 2(2,0)} \\
& H_{\overline{\overline{A 2(0,2)}}}^{\rightarrow} H_{A 2(0,2)} \\
& H_{\overline{B 0(0,0)}} \rightarrow H_{B 0(0,0)}+H_{B 1(1,0)}+H_{B 2(1,1)}+H_{B 2(0,0)} \\
& H_{\overline{B 1(0,1)}} \rightarrow H_{B 1(0,1)}+H_{B 2(0,2)} \\
& H_{\overline{B 2(1,1)}} \rightarrow H_{B 2(1,1)} \\
& H_{\overline{B 2(0,0)}} \rightarrow H_{B 2(0,0)}
\end{aligned}
$$

In terms (B7a-h), subscripts $i(j, k)$ stand now for the $i$-th order of Taylor' series expansion in nonadiabatic representation, while $j$ is the order of the coordinate operator $Q$ and the $k$ is the order of momentum operator $P$ within the given order of the Taylor's expansion.

The form of particular terms is,

$$
\begin{aligned}
& H_{A(0,0)}=E_{N N}^{0}\left(R_{0}\right)+\sum_{P Q} h_{P Q}^{0}\left(R_{0}\right) a_{P}^{+} a_{Q}+\frac{1}{2} \sum_{P Q R S} v_{P Q R S}^{0} a_{P}^{+} a_{Q}^{+} a_{S} a_{R} \\
& H_{A 1(1,0)}=\sum_{r} E_{N N}^{r} Q_{r}+\sum_{P Q r}\left[u_{P Q}^{r}+\sum_{R}\left(h_{P Q}^{0} c_{R Q}^{r}+h_{R Q}^{0} c_{R P}^{\check{r}^{*}}\right)\right] Q_{r} a_{P}^{+} a_{Q}+ \\
& +\sum_{P Q R S T r}\left(v_{P Q T S}^{0} c_{T R}^{r}+v_{T Q R S}^{0} c_{T P}^{\check{r}^{*}}\right) Q_{r} a_{P}^{+} a_{Q}^{+} a_{S} a_{R} \\
& H_{A 1(0,1)}=\sum_{r P Q R}\left(h_{P R}^{0} \hat{c}_{R Q}^{r}-h_{R Q}^{0} \hat{C}_{R P}^{r *}\right) P_{r} a_{P}^{+} a_{Q}+\sum_{r P Q R S T}\left(v_{P Q T S}^{0} \hat{c}_{T R}^{r}-v_{T Q R S}^{0} \hat{c}_{T P}^{r^{*}}\right) P_{r} a_{P}^{+} a_{Q}^{+} a_{S} a_{R} \\
& H_{A 2(1,1)}=\frac{1}{2} \sum_{r S P Q R}\left\{u_{P Q}^{r} \hat{C}_{R Q}^{s}-u_{R Q}^{r} \hat{C}_{R P}^{\breve{s}^{*}}+\sum_{S}\left[\left(h_{P R}^{0} c_{R S}^{r}+h_{R S}^{0} c_{R P}^{\check{r}^{*}}\right) \hat{c}_{S Q}^{s}-\left(h_{S R}^{0} C_{R Q}^{r}+h_{R Q}^{0} C_{R S}^{\check{r}^{*}}\right) \hat{c}_{S P}^{\breve{s}^{*}}\right]\right\} . \\
& \text {. }\left(Q_{r} P_{s}+P_{s} Q_{r}\right) a_{P}^{+} a_{Q}+
\end{aligned}
$$

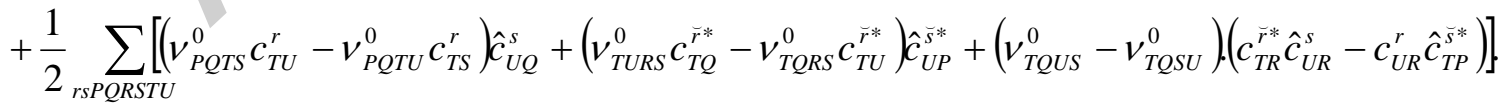

$$
\begin{aligned}
& \text {. }\left(Q_{r} P_{s}+P_{s} Q_{r}\right) a_{P}^{+} a_{Q}^{+} a_{S} a_{R}
\end{aligned}
$$




$$
\begin{aligned}
& H_{A 2(2,0)}=-\frac{1}{2} \sum_{r s} V_{N}^{r s} Q_{r} Q_{s}+ \\
& +\sum_{P Q r s}\left[\frac{1}{2} u_{P Q}^{r s}+\sum_{R}\left(\frac{1}{2} h_{P R}^{0} c_{R Q}^{r s}+\frac{1}{2} h_{R Q}^{0} c_{R P}^{\breve{r s} *}+u_{P R}^{r} c_{R Q}^{s}+u_{R Q}^{r} c_{R P}^{\breve{s}^{*}}\right)+\sum_{R S} h_{R S}^{0} c_{R P}^{\breve{r}^{*}} c_{S Q}^{s}\right] Q_{r} Q_{s} a_{P}^{+} a_{Q}+ \\
& +\frac{1}{2} \sum_{r S P Q R S T}\left\{v_{P Q T S}^{0} c_{T R}^{r s}+v_{T Q R S}^{0} c_{T P}^{\breve{r s} *}+\sum_{U}\left[v_{P Q T U}^{0} c_{T R}^{r} c_{U S}^{s}+v_{T U R S}^{0} c_{T P}^{\breve{r}^{*}} C_{U Q}^{\breve{s}^{*}}+2\left(v_{T Q U S}^{0}-v_{T Q S U}^{0}\right) c_{T P}^{\breve{r}^{*}} C_{U R}^{s}\right]\right\} . \\
& \text {. } Q_{r} Q_{S} a_{P}^{+} a_{Q}^{+} a_{S} a_{R} \\
& H_{A 2(0,2)}=-\frac{1}{2} \sum_{r s} W_{N}^{r s} P_{r} P_{s}+\sum_{r s P Q R}\left(\frac{1}{2} h_{P R}^{0} \hat{c}_{R Q}^{r s}+\frac{1}{2} h_{R Q}^{0} \hat{c}_{R P}^{r^{*} *}-\sum_{S} h_{R S}^{0} \hat{c}_{R P}^{r^{*}} \hat{c}_{S Q}^{s}\right) P_{r} P_{s} a_{P}^{+} a_{Q}+
\end{aligned}
$$

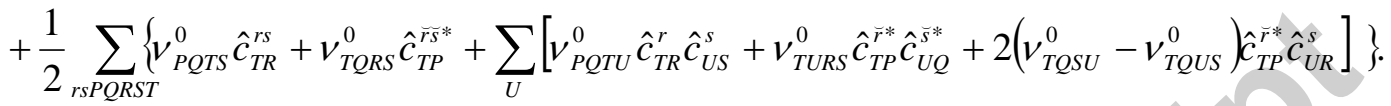

$$
\begin{aligned}
& \text {. } P_{r} P_{s} a_{P}^{+} a_{Q}^{+} a_{S} a_{R} \\
& H_{A 2(0,0)}=2 \sum_{r P Q} E_{N N}^{r} \hat{d}_{r P Q}^{0} a_{P}^{+} a_{Q}+ \\
& +\sum_{r P Q R}\left\{\left[u_{P R}^{r}+\sum_{S}\left(h_{P S}^{0} c_{S R}^{r}+h_{S R}^{0} c_{S P}^{r^{*}}\right)\right] \hat{d}_{r R Q}^{0}+\left[u_{R Q}^{r}+\sum_{S}\left(h_{R S}^{0} c_{S Q}^{r}+h_{S Q}^{0} c_{S R}^{r^{*}}\right)\right] \hat{d}_{r P R}^{0}\right\} . a_{P}^{+} a_{Q}+ \\
& +2 \sum_{r P Q R}\left[u_{P R}^{r}+\sum_{T}\left(h_{P T}^{0} c_{T R}^{r}+h_{T R}^{0} c_{T P}^{\check{r}^{*}}\right)\right] \hat{d}_{r Q S}^{0} a_{P}^{+} a_{Q}^{+} a_{S} a_{R}+ \\
& +\sum_{r P Q R S T U}\left[\left(v_{P Q T S}^{0} c_{T U}^{r}-v_{P Q T U}^{0} c_{T S}^{r}\right) \cdot \hat{d}_{r U Q}^{0}+\left(v_{T Q R S}^{0} c_{T U}^{\check{r}^{*}}-v_{T U R S}^{0} c_{T Q}^{\check{r}^{*}}\right) \cdot \hat{d}_{r P U}^{0}+\left(v_{T Q U S}^{0}-v_{T Q S U}^{0}\right)\left(c_{T P}^{\check{r}^{*}} \hat{d}_{r U R}^{0}+c_{U R}^{r} \hat{d}_{r P T}^{0}\right)\right] \\
& a_{P}^{+} a_{Q}^{+} a_{S} a_{R}+2 \sum_{r P Q R S T U V}\left(v_{P Q V T}^{0} c_{V S}^{r}+v_{V Q S T}^{0} c_{V P}^{\check{r}^{*}}\right) \cdot \hat{d}_{r R U}^{0} \cdot a_{P}^{+} a_{Q}^{+} a_{R}^{+} a_{U} a_{T} a_{S}
\end{aligned}
$$

$$
\begin{aligned}
& H_{B 0(0,0)}=\sum_{r} \hbar \omega_{r}\left(b_{r}^{+} b_{r}+\frac{1}{2}\right) \\
& H_{B 1(1,0)}=\sum_{r P Q} \hbar \omega_{r} \hat{d}_{r P Q}^{0} Q_{r} a_{P}^{+} a_{Q}
\end{aligned}
$$$$
H_{B 1(0,1)}=-\sum_{r P Q} \hbar \omega_{r} \hat{d}_{\breve{r} P Q}^{0} P_{r} a_{P}^{+} a_{Q}
$$$$
H_{B 2(1,1)}=\frac{1}{2} \sum_{r s P Q}\left(\hbar \omega_{r} \hat{d}_{r P Q}^{s}-\hbar \omega_{s} d_{\breve{s} P Q}^{r}\right)\left(Q_{r} P_{s}+P_{s} Q_{r}\right) \bar{a}_{P} \bar{a}_{Q}
$$$$
H_{B 2(2,0)}=0
$$$$
H_{B 2(0,2)}=-\sum_{r s P Q R} \hbar \omega_{r}\left(d_{\breve{r} P R}^{0} \hat{C}_{R Q}^{s}-d_{\breve{r} R Q}^{0} \hat{C}_{R P}^{\breve{s}^{*}}\right) P_{r} P_{s} a_{P}^{+} a_{Q}
$$$$
H_{B 2(0,0)}=\sum_{P Q R r} \hbar \omega_{r}\left(\hat{d}_{\breve{r} P R}^{0} \hat{d}_{r R Q}^{0}-d_{\breve{r} P R}^{0} d_{r R Q}^{0}\right) a_{P}^{+} a_{Q}+\sum_{P Q R S r} \hbar \omega_{r}\left(\hat{d}_{\breve{r} P R}^{0} \hat{d}_{r Q S}^{0}-d_{r P R}^{0} d_{r Q S}^{0}\right) a_{P}^{+} a_{Q}^{+} a_{S} a_{R}
$$

Nonadiabatic transformations (B1) preserve the total number of fermions (89) and Wick's theorem can be applied to all nonadiabatic terms with fermion operators. The result is normal 
product form with corresponding contractions. It enables SCF solution of the electronvibration problem. Also in this case after application of Wick's theorem, general set of all spinorbitals (P, Q, R, S) is divided on two groups, occupied spinorbitals (I,J) and unoccupied spinorbitals (A,B). Treatment of the particular transformed terms and solution of the problem is tedious but straightforward [33a].

Also in this case, the pure fermion part of the Hamiltonian (Wick's theorem is applied to all terms with fermion operators, but pure fermion part is represented only by terms without boson operators, i.e. B8a, B8g, B8n) is sum of three terms,

$H_{F(n a)}\left(R_{0}\right)=H_{F(n a)}^{0}\left(R_{0}\right)+H_{F(n a)}^{\prime}\left(R_{0}\right)+H_{F(n a)}^{\prime \prime}\left(R_{0}\right)$

a/ The ground state nonadiabatic total electronic energy is represented by zero-particle term (i.e. scalar quantity - contribution of contractions of fermion operators),

$H_{F(n a)}^{0}\left(R_{0}\right)=E_{0(c a d)}^{t e}\left(R_{0}\right)+\Delta E_{(n a)}^{0}=\left(E_{N N}^{0}\left(R_{0}\right)+E_{S C F}^{0}\left(R_{0}\right)\right)+\sum_{r A I} \hbar \omega_{r}\left(\left|c_{A I}^{r}\right|^{2}-\left|\hat{c}_{A I}^{2}\right|\right)$

As it is seen from (B10), the ground state crude-adiabatic total electronic energy $E_{0(\text { cad })}^{\text {te }}\left(R_{0}\right)$ at

fixed nuclear geometry $\mathrm{R}_{0}$ (contractions of $\mathrm{B} 8 \mathrm{a}$ ) is due to electron-vibration (phonon) coupling on the nonadiabatic level corrected by the difference of two terms,

$\Delta E_{(\text {na })}^{0}=-2 \sum_{r A I} \hbar \omega_{r}\left|\hat{c}_{A I}^{r}\right|^{2}+\sum_{r A I} \hbar \omega_{r}\left(\left|c_{A I}^{r}\right|^{2}+\left|\hat{c}_{A I}^{r}\right|^{2}\right)=\sum_{r A I} \hbar \omega_{r}\left(\left|c_{A I}^{r}\right|^{2}-\left|\hat{c}_{A I}^{r}\right|^{2}\right)$

This correction arises as contribution of the contractions of one and two-particle terms of (B8g) and (B8n). The form of (B10a) indicates that in principle, electronic ground state energy correction on nonadiabatic level $\Delta E_{(n a)}^{0}\left(R_{0}\right)$ can also be negative, in contrast to electronic ground state energy correction on adiabatic level (46, A30a), $\Delta E_{(a d)}^{0}\left(R_{0}\right)$, that is always positive. It depends on the value of nonadiabatic contributions $\hat{c}_{A I}^{r}$, i.e. on an extent of participation of the nuclear kinetic effect on electronic motion. In (B10a), summation runs over all normal modes (r), the unoccupied orbitals (A) and occupied orbitals (I).

b/ The one-particle term has the form,

$$
\begin{aligned}
& H_{F(n a)}^{\prime}\left(R_{0}\right)=\sum_{P} \varepsilon_{P(\text { cad })}^{0}\left(R_{0}\right) N\left[\bar{a}_{P}^{+} \bar{a}_{P}\right]-2 \sum_{r P Q} E^{r^{*}} \hat{c}_{P Q}^{r} N\left[a_{P}^{+} a_{Q}\right]+ \\
& +\sum_{r P Q} \hbar \omega_{r}\left(\sum_{A}\left(c_{P A}^{r} c_{Q A}^{r *}-\hat{c}_{P A}^{r} \hat{c}_{Q A}^{r *}\right)-\sum_{I}\left(c_{P I}^{r} c_{Q I}^{r *}-\hat{c}_{P I}^{r} \hat{c}_{Q I}^{r *}\right)\right) N\left[a_{P}^{+} a_{Q}\right]+ \\
& +\sum_{r P Q}\left((h(P)-p(P)) \varepsilon_{P}^{r *}+(h(Q)-p(Q)) \varepsilon_{Q}^{r^{*}}\right) \hat{c}_{P Q}^{r} \cdot N\left[a_{P}^{+} a_{Q}\right]- \\
& -\sum_{r P Q A I}\left(\left(v_{P I Q A}^{r}-v_{P I A Q}^{r}\right) \hat{c}_{I A}^{r *}+\left(v_{P A Q I}^{r}-v_{P A Q}^{r}\right) \cdot \hat{c}_{A I}^{r *}\right) \cdot N\left[a_{P}^{+} a_{Q}\right]
\end{aligned}
$$

In (B11) shorthand notation has been used,

$$
v_{P Q R S}^{r}=\sum_{T}\left(v_{P Q T S}^{0} c_{T R}^{r}+v_{P Q R T}^{0} c_{T S}^{r}-v_{T Q R S}^{0} c_{P T}^{r}-v_{P T R S}^{0} c_{Q T}^{r}\right)
$$

For symbols $h, p$, holds; $h(A)=0, h(I)=1, p(A)=1, p(I)=0$.

As it is seen from (B11), this part represents electronic spectrum of the system corrected (all terms following after the first, crude adiabatic, term) by nonadiabatic electronvibration(phonon) interactions. 
c/ The two-particle fermion part of the Hamiltonian has the form,

$$
\begin{aligned}
& H_{F(n a)}^{\prime \prime}\left(R_{0}\right)=\frac{1}{2} \sum_{P Q R S} v_{P Q R S(c a d)}^{0} N\left[\bar{a}_{P}^{+} \bar{a}_{Q}^{+} \bar{a}_{S} \bar{a}_{R}\right]+ \\
& +\sum_{r P Q R S} \hbar \omega_{r}\left(c_{P R}^{r} c_{S Q}^{r *}-\hat{c}_{P R}^{r} \hat{c}_{S Q}^{r *}\right) N\left[a_{P}^{+} a_{Q}^{+} a_{S} a_{R}\right]- \\
& -2 \sum_{r P Q S} \varepsilon_{P}^{r} \hat{c}_{S Q}^{r^{*}} N\left[a_{P}^{+} a_{Q}^{+} a_{S} a_{P}\right]+ \\
& +\sum_{r P Q R S T} \sum_{I}\left[v_{P Q T S}^{0} c_{T I}^{r}-v_{P Q T I}^{0} c_{T S}^{r}+\left(v_{T Q S I}^{0}-v_{T Q I S}^{0}\right) c_{P T}^{r}\right] \hat{c}_{R I}^{r^{*}} N\left[a_{P}^{+} a_{Q}^{+} a_{S} a_{R}\right]+ \\
& +\sum_{r P Q R S T} \sum_{I}\left[v_{T I R S}^{0} c_{Q T}^{r}-v_{T Q R S}^{0} c_{I T}^{r}+\left(v_{I Q T S}^{0}-v_{I Q S T}^{0}\right) c_{T R}^{r}\right] \hat{c}_{I P}^{r^{*}} N\left[a_{P}^{+} a_{Q}^{+} a_{S} a_{R}\right]- \\
& -\sum_{r P Q R S T} \sum_{A}\left[v_{P Q T S}^{0} c_{T A}^{r}-v_{P Q T A}^{0} c_{T S}^{r}+\left(v_{T Q S A}^{0}-v_{T Q A S}^{0}\right) c_{P T}^{r}\right] \hat{c}_{R A}^{r *} N\left[a_{P}^{+} a_{Q}^{+} a_{S} a_{R}\right]- \\
& -\sum_{r P Q R S T} \sum_{A}\left[v_{T A R S}^{0} c_{Q T}^{r}-v_{T Q R S}^{0} c_{A T}^{r}+\left(v_{A Q T S}^{0}-v_{A Q S T}^{0}\right) c_{T R}^{r}\right] \hat{c}_{A P}^{r *} N\left[a_{P}^{+} a_{Q}^{+} a_{S} a_{R}\right]
\end{aligned}
$$

This two-particle term represents electron correlation energy corrected (all terms following after the first, crude adiabatic, term) by nonadiabatic electron-vibration(phonon) interactions.

The pure fermion part of the Hamiltonian (B9, B10, B11, B12) does not depend on boson operators. It means that this part of the Hamiltonian is invariant of vibration (phonon) excitations. However, besides the terms B8a,g,n that constitute pure fermion part, all other terms, i.e. B8b,c,d,e,f,h,i,j,k,m depend on boson operators. These terms constitute an effective fermion Hamiltonian that depends on boson excitations. For boson vacuum $|0\rangle$, the effective part is written as $H_{\langle F\rangle}$,

$H_{\langle F\rangle}=\langle 0|B 8 b+B 8 c+B 8 d+B 8 e+B 8 f+B 8 h+B 8 i+B 8 j+B 8 m| 0\rangle$

Integration over boson operators and application of Wick's theorem on fermion operators divides (B13) on the sum of three terms,

$H_{\langle F\rangle}=H_{\langle F\rangle}^{0}+H_{\langle F\rangle}^{\prime \prime}+H_{\langle F\rangle}^{\prime \prime}$

a/ Effective zero-particle term represents vibration zero-point energy, i.e.

$H_{\langle F\rangle}^{0}=\frac{1}{2} \sum_{r} \hbar \omega_{r}$

This term and term $B(10)$ represent total energy of the system that account for nonadiabatic electron-vibration(phonon) interactions in its electronic ground state at boson vacuum. b/ Effective one-particle term has the form,

$$
\begin{aligned}
& H_{\langle F\rangle}^{\prime}=\frac{1}{2} \sum_{r P} u_{P P}^{r r *} N\left[a_{P}^{+} a_{P}\right]+ \\
& +\sum_{r P R}\left(\left(\varepsilon_{P}^{0}-\varepsilon_{R}^{0}\right)\left(\left|c_{P R}^{r}\right|^{2}+\left|\hat{c}_{P R}^{r}\right|^{2}\right)-2 \hbar \omega_{r} \operatorname{Re}\left(\hat{c}_{P R}^{r} c_{P R}^{r *}\right)\right) N\left[a_{P}^{+} a_{P}\right]+ \\
& +\sum_{r P A I}\left(\sum_{R}\left(v_{P R P A}^{0}-v_{P R A P}^{0}\right)\left(c_{I R}^{r} c_{I A}^{r *}+\hat{c}_{I R}^{r} \hat{c}_{I A}^{r *}\right)-\left(v_{P R P I}^{0}-v_{P R I P}^{0}\right)\left(c_{A R}^{r} c_{A I}^{r *}+\hat{c}_{A R}^{r} \hat{c}_{A I}^{r *}\right)\right) N\left[a_{P}^{+} a_{P}\right]+ \\
& +\sum_{r P A I}\left(\frac{1}{2} \sum_{R}\left(v_{P I P A}^{0}-v_{P I A P}^{0}\right)\left(c_{A I}^{r \check{r}}-\hat{c}_{A I}^{r \check{r}}\right)-\left(v_{P A P I}^{0}-v_{P A I P}^{0}\right)\left(c_{I A}^{r \breve{r}}-\hat{c}_{I A}^{r \breve{r}}\right)\right) N\left[a_{P}^{+} a_{P}\right]
\end{aligned}
$$


c/ Form of the effective two-particle term is,

$$
\begin{aligned}
& H_{\langle F\rangle}^{\prime \prime}=\frac{1}{2} \sum_{r P Q R S T}\left(v_{P Q T S}^{0}\left(c_{T R}^{r \breve{r}}-\hat{c}_{T R}^{r \check{r}}\right)+v_{T Q R S}^{0}\left(c_{T P}^{r \check{r} *}-\hat{c}_{T P}^{r^{*} \breve{r}}\right)\right) N\left[a_{P}^{+} a_{Q}^{+} a_{S} a_{R}\right]-
\end{aligned}
$$

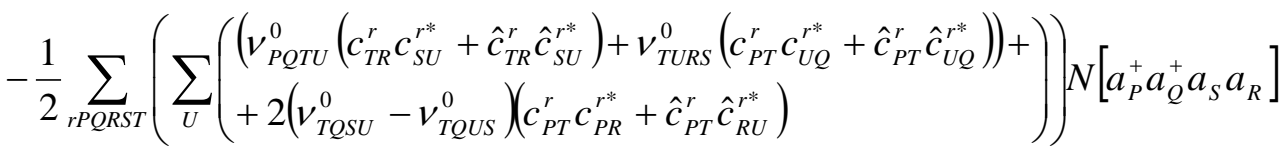

The equations that hold for coefficients of transformation matrices $C$ and $\hat{C}$ can be derived from one-particle terms in first order of Taylor's expansion in boson operators, i.e. from terms B8b,c,i and B8j after Wick's theorem application. Diagonalization imposed on these terms, i.e.

$$
H_{1(1,0)}^{\prime}=\sum_{r P} \varepsilon_{P}^{r} Q_{r} N\left[a_{P}^{+} a_{P}\right], \quad H_{1(0,1)}^{\prime}=\sum_{r P} \hat{\varepsilon}_{P}^{r} P_{r} N\left[a_{P}^{+} a_{P}\right]
$$

results in coupled equations for transformation coefficients $c_{P Q}^{r}$ and $\hat{c}_{P Q}^{r}$;

$$
\begin{aligned}
& u_{P Q}^{r}+\left(\varepsilon_{P}^{0}-\varepsilon_{Q}^{0}\right) c_{P Q}^{r}+\sum_{A I}\left[\left(v_{P I Q A}^{0}-v_{P I A Q}^{0}\right) c_{A I}^{r}-\left(v_{P A Q I}^{0}-v_{P A I}^{0}\right) c_{I A}^{r}\right]-\hbar \omega_{r} \hat{c}_{P Q}^{r}=\varepsilon_{P}^{r} \delta_{P Q} \\
& \left(\varepsilon_{P}^{0}-\varepsilon_{Q}^{0}\right) \hat{c}_{P Q}^{r}+\sum_{A I}\left[\left(v_{P I Q A}^{0}-v_{P I A Q}^{0}\right) \hat{c}_{A I}^{r}-\left(v_{P A Q I}^{0}-v_{P A I Q}^{0}\right) \hat{c}_{I A}^{r}\right]-\hbar \omega_{r} c_{P Q}^{r}=\hat{\varepsilon}_{P}^{r} \delta_{P Q}
\end{aligned}
$$

Solution of diagonalization equations yields off-diagonal adiabatic transformation coefficients $c_{P Q}^{r}$ and off-diagonal nonadiabatic transformation coefficients $\hat{c}_{P Q}^{r}, P \neq Q$. For diagonal elements holds $c_{P P}^{r}=\hat{c}_{P P}^{r}=0$ (see A7, B5; $c_{P Q}^{0}=\hat{c}_{P Q}^{0}=\delta_{P Q}$ ). In adiabatic limit when $\hat{c}=0$, and $\hbar \omega_{r}<<\left|\varepsilon_{P}^{0}-\varepsilon_{Q}^{0}\right|$, eq. B16b can be omitted and adiabatic CPHF equations (A33) are recovered. In this context, coupled set of equations (B16a, B16b) represents generalized CPHF (GCPHF) equations.

From zero-particle terms (these arise as contractions after Wick's theorem application) in second-order of Taylor's expansion in $Q$ (B8e, B8l) or in $P$ (B8f, B8m) respectively, for corrections to potential energy $V_{N}^{r s}$ and for corrections to nuclear kinetic energy $W_{N}^{r s}$ can be derived,

$V_{N}^{r s}=\sum_{I} u_{I I}^{r s}+\sum_{A I}\left[\left(u_{I A}^{r}+\hbar \omega_{r} \hat{c}_{I A}^{r}\right) c_{A I}^{s}+\left(u_{I A}^{s}+\hbar \omega_{r} \hat{C}_{I A}^{s}\right) c_{A I}^{r}\right]$

$W_{N}^{r s}=2 \hbar \omega_{r} \sum_{A I} c_{A I}^{r} \hat{c}_{I A}^{s}$

These corrections modify nuclear potential and nuclear kinetic energy, i.e. $E_{\text {pot }}(\bar{Q})=E_{N N}^{(2)}(\bar{Q})+V_{N}^{(2)}(\bar{Q}), E_{k i n}(\bar{P})=T_{N}(\bar{P})+W_{N}^{(2)}(\bar{P})$ that are used at solution of secular equations of nuclear motion - see (A35). In the adiabatic limit $\hat{c}=0$, correction to potential energy is reduced to adiabatic expression (A34) and kinetic energy correction equals zero. Term $W_{N}^{r s}$ is different from zero only if nonadiabatic effect cannot be neglected, i.e. in case when system is in antiadiabatic state and inequality $\left|\varepsilon_{I}^{0}(R)-\varepsilon_{A}^{0}(R)\right| \leq \hbar \omega_{r}$ holds.

Appendix C: Approximate solution of GCPHF equations

Knowledge of the matrix elements $c$ and $\hat{c}$ of transformation matrices enables calculation of all corrections to nonadiabatic Q,P-dependent form of electronic Hamiltonian that are consequence of electron-vibration (phonon) interactions. The exact treatment requires iterative solution of diagonalization equations that are represented by coupled set of GCPHF equations (B16a,b) and secular equations of nuclear motion (A35) for particular fixed nuclear configuration $R_{0}$. If it is accepted that electron-vibration(phonon) interactions influence 
system Hamiltonian mainly over one-electron term, as it is general treatment in solid-state theory (see the form of interaction term (39b) and also (1) in the present paper), then an approximate solution of GCPHF equations (B16a,b) can be obtained. In this case, it is reasonable to neglect contributions of two-electron terms in GCPHF equations (B16a,b). The diagonalization equations are then of the approximate form (C1a,b),

$$
\begin{aligned}
& u_{P Q}^{r}+\left(\varepsilon_{P}^{0}-\varepsilon_{Q}^{0}\right) c_{P Q}^{r}-\hbar \omega_{r} \hat{c}_{P Q}^{r}=\varepsilon_{P}^{r} \delta_{P Q} \\
& \left(\varepsilon_{P}^{0}-\varepsilon_{Q}^{0}\right) \hat{c}_{P Q}^{r}-\hbar \omega_{r} c_{P Q}^{r}=\hat{\varepsilon}_{P}^{r} \delta_{P Q}
\end{aligned}
$$

With respect to calibration for diagonal matrix elements, $c_{P P}^{r}=\hat{c}_{P P}^{r}=0$, solution of approximate diagonalization equations $(\mathrm{C} 1 \mathrm{a}, \mathrm{b})$ for off-diagonal matrix elements can be derived in the analytic form,

$$
\begin{aligned}
& c_{P Q}^{r}=u_{P Q}^{r} \frac{\left(\varepsilon_{P}^{0}-\varepsilon_{Q}^{0}\right)}{\left(\hbar \omega_{r}\right)^{2}-\left(\varepsilon_{P}^{0}-\varepsilon_{Q}^{0}\right)^{2}} ; P \neq Q \\
& \hat{c}_{P Q}^{r}=u_{P Q}^{r} \frac{\hbar \omega_{r}}{\left(\hbar \omega_{r}\right)^{2}-\left(\varepsilon_{P}^{0}-\varepsilon_{Q}^{0}\right)^{2}} ; P \neq Q
\end{aligned}
$$

Two extreme limits are interesting;

a/ strong adiabatic regime, $\hbar \omega_{r} /\left|\varepsilon_{P}^{0}-\varepsilon_{Q}^{0}\right| \rightarrow 0$. In this case,

$$
c_{P Q}^{r}=\frac{u_{P Q}^{r}}{\left(\varepsilon_{Q}^{0}-\varepsilon_{P}^{0}\right)} \text {, and } \hat{c}_{P Q}^{r}=0
$$

b/ strong nonadiabatic regime, $\hbar \omega_{r} /\left|\varepsilon_{P}^{0}-\varepsilon_{Q}^{0}\right| \rightarrow \infty$. In this case,

$$
c_{P Q}^{r}=0 \text {, and } \hat{c}_{P Q}^{r}=\frac{u_{P Q}^{r}}{\hbar \omega_{r}}
$$

For an intermediate regime, both transformation coefficients contribute to energy corrections. The limit, $\hbar \omega_{r} /\left|\varepsilon_{P}^{0}-\varepsilon_{Q}^{0}\right| \rightarrow 1$, would result in singularities of $(\mathrm{C} 2 \mathrm{a}, \mathrm{b})$ but this case can be

excluded since for transformation coefficients have to hold $\left|c_{P Q}^{r}\right|<1>\left|\hat{c}_{P Q}^{r}\right|$ with respect to validity of the Taylor' series expansions (A4, B4).

\section{References}

[1] J. G. Bednorz and K. A. Muller, Z. Phys. B: Cond. Matter 64 (1986) 189

[2] P. H. Hor, R. L. Meng, Y. Q. Wang, L. Gao, Z. J. Hung, J. Bechtold, K. Forster, and C. W. Chu, Phys. Rev. Lett. 58 (1987) 1191

[3] J. Bardeen, L. N. Cooper and J. R. Schrieffer, Phys. Rev. 108 (1957) 1175

[4] A. B. Migdal, Zh. Eksp. Teor. Fiz. 34 (1958) 1438; Sov. Phys. JETP 7 (1958) 996

[5] G. M. Eliashberg, Zh. Eksp. Teor. Fiz. 38 (1960) 996; 39 (1960) 1437; Sov. Phys. JETP 11 (1960) 696; 12 (1960) 1000

[6] A.S. Alexandrov, Physica C 363 (2001) 231

[7] see e.g., P. W. Anderson, The theory of superconductivity in high- $\mathrm{T}_{\mathrm{c}}$ cuprates (Princeton University Press, Princeton) (1997)

[8] E. Dagotto E., Rev.Modern Phys. 66 (1994) 763

[9] Th. Maier, M.Jarrell, T.Pruschke and J.Keller, Phys. Rev. Lett. 85 (2000) 1524

[10] S. Sorella, G.B.Martins, F.Becca, C.Gazza, L.Capriotti,A.Parola and E.Dogotto, Phys.Rev.Lett. 88 (2001) 117002

[11] S. R. White and D. Scalapino, Phys. Rev. Lett. 80 (1998) 1272; Phys. Rev. B 60 (1999)753 
[12] A. Paramekanti, M. Randeria and N Trivedi, Phys. Rev. B 70 (2004) 054504

[13] A. Lanzara, P. V. Bogdanov, X. J. Zhou, S.A.Kellar, D.L.Feng, E.D.Lu, T.Yoshida, H.Eisaki, A.Fujimori, K.Kishio, J.I. Shimoyama, T.Noda, S.Uchida, Z.Hussain and Z.X.Shen, Nature 412 (2001) 510

[14] X. J. Zhou, T.Yoshida, A. Lanzara, P.V.Bogdanov, S.A.Kellar, K.M.Shen, W.L.Yang, F.Ronning, T.Sasagawa, T.Kakeshita, T.Noda, H.Eisaki, S.Uchida, C.T.Lin, F.Zhou, J.W.Xiong, W.X.Ti, Z.X.Zhao, A.Fujimori, Z.Hassain and Z.X.Shen, Nature 423 (2003) 398

[15] T.Takahashi, T. Sato, H. Matsui and K.Terashima, New J. Phys. 7 (2005) 105

[16] J. H. Chung, T. Egami, R. J. McQuinney, M.Yethiraj, M.Arai, T.Yokoo,Y.Petrov, H.A.Mook, Y.Endoch, S.Tajima, C.Frost and F.Dogan, Phys. Rev. B 67 (2003) 014517

[17] L. Pintschovius, D. Reznik, W. Reichardt, Y.Endoch, H.Hiraka, J.M.Tranquada, H.Uchiama, T.Masui, and S.Tajima, Phys. Rev. B 69 (2004) 214506

[18] a/L. Pietronero, Europhys. Lett. 17 (1992) 365;

b/L. Pietronero and S. Strassler, Europhys. Lett. 18 (1992) 627;

[19] L. Proville and S. Aubry, Eur.Phys.J. B 11 (1999) 41

[20] J. Bonca, S.A. Trugman and I. Batistic, Phys.Rev. B 60 (1999) 1633

[21] P. Benedetti and R. Zeyher, Phys. Rev. B 58 (1998) 14320

[22] H. Fehske, J. Loos and G. Wellein, Z. Phys. B 104 (1997) 619

[23] A.R. Bishop and M. Salkola, in Polarons and Bipolarons, in High-Tc Superconductors and Related materials, Ed. E.K.H. Salje (Cambridge University Press, Cambridge (1995)

[24] a/A.S. Alexandrov and P.E. Kornilovitch, J. Supercond. 15 (2002) 403

b/A.S. Alexandrov and J. Runninger, Phys. Rev. B 23 (1981) 1796

c/A.S. Alexandrov and J. Runninger, Phys. Rev. B 24 (1981) 1164

d/A.S. Alexandrov, Zh.Fiz.Chim 57 (1983) 273 (Rus. J.Phys.Chem. 57 (1983) 167

[25] J.M.An and W.E. Picket, Phys.Rev.Lett. 86 (2001) 4366

[26] H.Rosner, J.M.An, W.E.Picket, S.L.Drechsler, Phys.Rev.B 66 (2002) 024521

[27] T.Yilderim, O.Gulseren, J.W.Lynn, C.M.Brown, T.J.Udovic, Q.Huang, N.Rogado, K.A.Regan, M.A.Hayward, J.S.Slusky, T.He, M.K.Hass, P.Khalifah, K.Inumaru and R.J.Cava, Phys.Rev.Lett. 87 (2001) 037001

[28] P.Banacky, Int.J.Quant.Chem. 101 (2005) 131

[29] L.Boeri, E.Cappelluti and L.Pietronero, Phys.Rev.B 71 (2005) 012501

[30] L.Pietronero, S.Strassler and C.Grimaldi, Phys.Rev.B 52 (1995) 10516

[31] P.Banacky, Physica C 460-462 (2007) 1115

[32] E.Capelluti and L.Pietronero, J.Phys.Chem.Solids 67 (2006) 1941

[33] M.Svrcek, P.Banacky and A.Zajac, Int.J.Quant.Chem. a/43 (1992) 393; b/43 (1992) 415 ; c/43 (1992) 425; d/43 (1992) 551

[34] M.Born and J.R.Oppenheimer, Ann.Phys. 84 (1927) 457

[35] M.Born and K.Huang, Dynamical theory of Crystal Lattices (London, Oxford University Press, 1956)

[36] A.S.Davydov, Quantum Mechanics (New York, Addison-Wesley, 1965)

[37] P. Pulay, in Application of Electronic structure Theory, Modern Theoretical Chemistry (Plenum Press, New York, 1997), Vol.4

[38] J.Gerrat and I.M.Mills, J.Chem.Phys. 49 (1968) 1719; 49 (1968) 1730

[39] J.A.Pople, R.Krishnan, H.B.Schlegel and J.S.Binkley, Int.J.Quant.Chem. Symp.13 (1979) 225

[40] I.Hubac and M.Svrcek, Int.J.Quant.Chem. 33 (1988) 403

[41] M.Svrcek, P.Banacky, S.Biskupic, J.Noga, P.Pelikan and A.Zajac, Chem.Phys.Lett. 299 (1999) 151

[42] W.Kutzelnigg, Mol.Phys. 90 (1997) 909

[43] N.C.Handy, A.M.Lee, Chem.Phys.Lett. 252 (1996) 425

[44] N.C.Handy, Y.Yamaguchi and H.F.Schaeffer III, J.Chem.Phys. 84 (1986) 4481

[45] W.Kolos, W.Wolniewicz, J.Chem.Phys. 41 (1964) 3663

[46] W.Wolniewicz, J.Chem.Phys. 99 (1993) 1851

[47] M.Wagner, Unitary Transformations in Solid-State Physics. Modern Problems in Condensed Matter Sciences, vol.15 (North-Holland, New York, 1986)

[48] T.D.Lee, F.Low, D.Pines, Phys.Rev. 90 (1953) 297 